\title{
Photodynamical analysis of the triply eclipsing hierarchical triple system EPIC 249432662
}

T. Borkovits ${ }^{\oplus}, 1,2 \star$ S. Rappaport, ${ }^{3}$ T. Kaye,${ }^{4}$ H. Isaacson, ${ }^{5}$ A. Vanderburg, ${ }^{6,7} \dagger$

A. W. Howard, ${ }^{8}$ M. H. Kristiansen,,${ }^{9,10}$ M. R. Omohundro $\ddagger$ H. M. Schwengeler $\ddagger$ I. A. Terentev, $\ddagger$ A. Shporer,${ }^{11}$ H. Relles, ${ }^{11}$ S. Villanueva Jr, ${ }^{11,12}$ T. G. Tan, ${ }^{13}$ K. D. Colón, ${ }^{14}$ J. Blex, ${ }^{15}$ M. Haas, ${ }^{15}$ W. Cochran ${ }^{7}$ and M. Endl ${ }^{7}$

${ }^{1}$ Baja Astronomical Observatory of Szeged University, H-6500 Baja, Szegedi út, Kt. 766, Hungary

${ }^{2}$ Konkoly Observatory, Research Centre for Astronomy and Earth Sciences, Hungarian Academy of Sciences, Konkoly Thege Miklós út 15-17, H-1121 Budapest, Hungary

${ }^{3}$ Department of Physics, Kavli Institute for Astrophysics and Space Research, M.I.T., Cambridge, MA 02139, USA

${ }^{4}$ Raemor Vista Observatory, 7023 E. Alhambra Dr., Sierra Vista, AZ 85650, USA

${ }^{5}$ Department of Astronomy, University of California at Berkeley, Berkeley, CA 94720-3411, USA

${ }^{6}$ Harvard-Smithsonian Center for Astrophysics, 60 Garden Street, Cambridge, MA 02138 USA

${ }^{7}$ Department of Astronomy, The University of Texas at Austin, 2515 Speedway, Stop C1400, Austin, TX 78712

${ }^{8}$ Astronomy Department, California Institute of Technology, MC 249-17, 1200 E. California Blvd, Pasadena, CA 91125, USA

${ }^{9}$ DTU Space, National Space Institute, Technical University of Denmark, Elektrovej 327, DK-2800 Lyngby, Denmark

${ }^{10}$ Brorfelde Observatory, Observator Gyldenkernes Vej 7, DK-4340 Tфllфse, Denmark

${ }^{11}$ Kavli Institute for Astrophysics and Space Research, M.I.T., Cambridge, MA 02139, USA

${ }^{12}$ Department of Astronomy, The Ohio State University, Columbus, OH 43210, USA

${ }^{13}$ Perth Exoplanet Survey Telescope, Perth, Western Australia 6010, Australia

${ }^{14}$ NASA Goddard Space Flight Center, Exoplanets and Stellar Astrophyscs Laboratory (Code 667), Greenbelt, MD 20771, USA

${ }^{15}$ Astronomisches Institut, Ruhr Universität, 44780 Bochum, Germany

Accepted 2018 November 20. Received 2018 November 20; in original form 2018 September 3

\begin{abstract}
Using Campaign 15 data from the $K 2$ mission, we have discovered a triply eclipsing triple star system: EPIC 249432662. The inner eclipsing binary system has a period of $8.23 \mathrm{~d}$, with shallow $\sim 3$ per cent eclipses. During the entire 80-d campaign, there is also a single eclipse event of a third body in the system that reaches a depth of nearly 50 percent and has a total duration of $1.7 \mathrm{~d}$, longer than for any previously known third-body eclipse involving unevolved stars. The binary eclipses exhibit clear eclipse timing variations. A combination of photodynamical modeling of the light curve, as well as seven follow-up radial velocity measurements, has led to a prediction of the subsequent eclipses of the third star with a period of $188 \mathrm{~d}$. A campaign of follow-up ground-based photometry was able to capture the subsequent pair of third-body events as well as two further 8-d eclipses. A combined photospectro-dynamical analysis then leads to the determination of many of the system parameters. The 8-d binary consists of a pair of M stars, while most of the system light is from a K star around which the pair of $\mathrm{M}$ stars orbits.
\end{abstract}

Key words: binaries: close-binaries: eclipsing-stars: individual: EPIC 249432662.

\section{INTRODUCTION}

Hierarchical triple stellar systems and/or subsystems form a small but very important subgroup of the zoo of multiple stellar systems.

^E-mail: borko@electra.bajaobs.hu

$\dagger$ NASA Sagan Fellow.

$\ddagger$ Citizen Scientist.
Their significance in the formation of the closest main-sequence binary systems (see e.g. Eggleton \& Kiseleva-Eggleton 2001; Fabrycky \& Tremaine 2007; Naoz \& Fabrycky 2014; Maxwell \& Kratter 2018, and further references therein) is widely acknowledged. Hierarchical triples have also been hypothetized to play a significant role in the formation of some kinds of peculiar objects, such as blue stragglers (Perets \& Fabrycky 2009), low-mass X-ray binaries (Shappee \& Thompson 2013), and some peculiar binary pulsars 


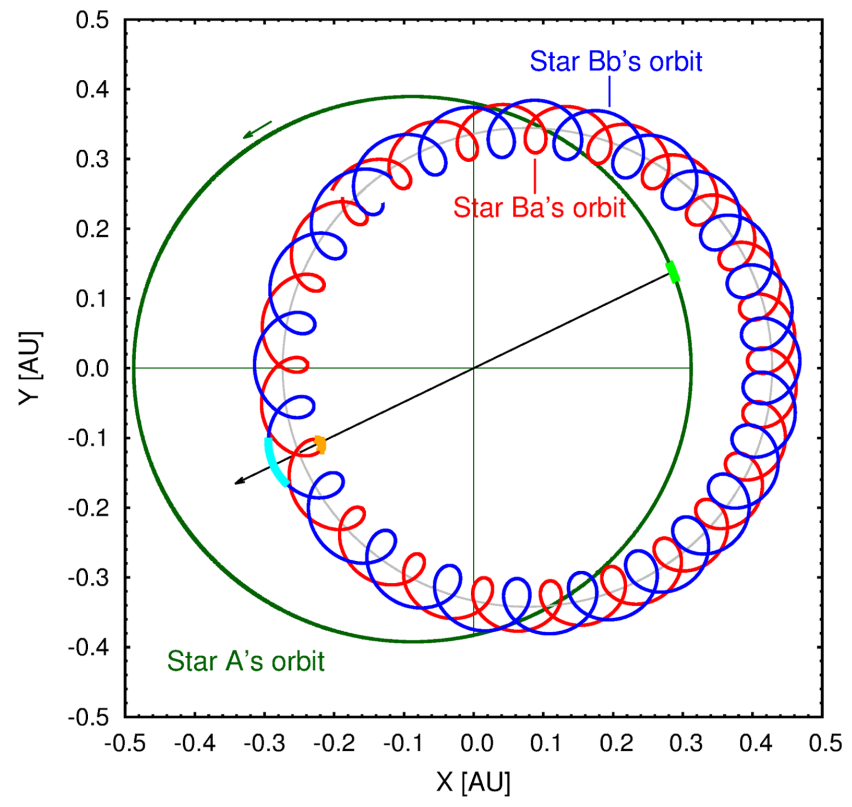

Figure 1. Pole-on view of the hierarchical triple star system EPIC 249432662. The red and blue curves represent the motions of the $\mathrm{Ba}$ and $\mathrm{Bb}$ stars of the 'inner' 8-d binary orbit in their 188-d 'outer' orbit about the center of mass $(\mathrm{CM})$ of the triple system (located at $X=Y=0$ ). The thin grey curve marks the locus of $\mathrm{CM}$ points for the 8-d binary. The green curve is the 188-d 'outer' orbit of star A, the third star that comprises the system. The thin green lines denote the major and minor axes of the orbit of star A, while the thin green arrow indicates the direction of motion along the orbits. Thicker sections of the orbits represent the arcs on which the three stars were moving during the 'great eclipse', observed with the Kepler spacecraft around BJD 2458018 . The black arrow that connects these arcs is directed toward the observer.

(see e.g. Portegies Zwart et al. 2011). They might even have a role in driving two white dwarfs to merger in that scenario for type Ia supernova explosions (Maoz, Mannucci \& Nelemans 2014).

In the case of hierarchical triples, one of the three mutual distances among the three components of the system remains substantially smaller than the other two distances for the entire lifetime of the triple. Therefore, the dynamics of the triple can be well approximated with the (slightly perturbed) Keplerian motions of two 'binaries': an inner or close binary, formed by the two components having the smallest separation, and an outer or wide 'binary', consisting of the centre of mass of the inner pair and the more distant third object (see Fig. 1 for a schematic diagram). ${ }^{1}$ For most of the known hierarchical triple star systems, with large period ratios for the 'outer binary' to 'inner binary', departures from pure Keplerian motion are expected to become significant only on time-scales of decades, centuries, or even millenia, i.e. over much longer intervals than the length of the available observational data trains. However,

\footnotetext{
${ }^{1}$ In this paper, we use the following notations. The orbital elements of the inner and outer orbits are subscripted by numbers ' 1 ' and ' 2 ', respectively. Regarding the three stars, we label them as $A, B a$, and $B b$, where $A$ denotes the brightest and most massive component, i.e. the distant, third star, while $B a$ and $B b$ refer to the primary and the secondary components of the close, inner 8-d binary. When we refer to physical quantities of individual stars, we use these subscripts. In such a way, for example, $m_{\mathrm{A}}$ or $m_{\mathrm{Ba}}$ stands for the masses of components $A$ or $B a$, respectively, but $m_{\mathrm{B}}$ denotes the total mass of the inner binary, $\left(m_{\mathrm{Ba}}+m_{\mathrm{Bb}}\right)$, while $m_{\mathrm{AB}}$ refers to the total mass of the entire triple system.
}

there is an important subgroup of the hierarchical triple systems, the so-called 'compact hierarchical triples' (CHT), which have smaller ratios of 'outer' to 'inner' periods and, occasionally, also smaller characteristic orbital dimensions. These may show much shorter time-scale and well-observable dynamical (or other kinds of) interactions so that they allow us to promptly determine many of the important dynamical and astrophysical parameters of these systems.

For example, a careful analysis based (partly) on the dynamically perturbed pulsar timing data of the millisecond pulsar PSR J0337+1715 orbiting in a peculiar CHT consisting of two white dwarfs in addition to the pulsar component has led to the accurate determination of the masses of all three objects, as well as the spatial configuration of the triple (Ransom, Stairs \& Archibald 2014). Similarly, as shown by Borkovits et al. (2011, 2015), if the close pair of a CHT happens to be an eclipsing binary (EB), the dynamical perturbations of the third companion on the orbital motion of the EB manifest themselves in intensive and quasi-cyclic eclipse timing variations (ETVs) on the time-scale of the orbital period of the outer component. The analysis of this effect makes it possible to determine not only the complete spatial configuration of the triple system but also the masses of the three objects.

As an application of the latter ETV analysis method, Borkovits et al. (2016) investigated 62 such CHTs in the original field of the Kepler space telescope (Borucki, Koch \& Basri 2010) where the inner binary was an EB, and the dynamical interactions were significant. They were able to determine many of the system parameters, including the mutual inclination of the planes of the inner and outer orbits, which is a key parameter from the point of view of the different triple star formation theories (see e.g. Tokovinin 2017). In the same paper the authors identified an additional 160 CHTs through the traditional light-travel time effect (LTTE; see e.g. Irwin 1959) and, in total, they found $\sim 104$ CHTs with outer orbital period $P_{2} \lesssim 1000 \mathrm{~d}$; this provides by far the most populated sample of hierarchical triple stars at the lower end of their outer period distribution. They pointed out a significant dearth of ternaries with $P_{2} \lesssim 200 \mathrm{~d}$, and concluded that this fact cannot be explained with observational selection effects. This latter result is in accord with the previous findings of Tokovinin et al. (2006) on the unexpected rarity of (the mostly spectroscopically discovered) triple systems in the period regime $P_{2}<1000 \mathrm{~d}$, whose shortage is more explicit amongst those CHTs which contain exclusively solar mass and/or less massive components (Tokovinin 2014). Therefore, investigations of such systems are especially important.

A very narrow subgroup of CHTs that offers further extraordinary possibilities for accurate system parameter determinations are those triples, which exhibit outer eclipses. These systems have a fortuitous orientation of the triple system relative to the observer whereby, occasionally, the distant third component eclipses one or both stars of the inner close binary or, vice versa, it is eclipsed by them. Such phenomena had never been seen before the advent of the Kepler era. The Kepler space telescope's 4yr-long, quasi-continuous observations, made with unprecedented photometric precision have, however, led to the discovery of at least 11 CHTs exhibiting outer eclipses, and a similar number of circumbinary transiting extrasolar planets. The latter group, though dynamically similar, are not considered in the following list of CHTs. These are KIC 05897826 (= KOI-126; Carter, Fabrycky \& Ragozzine 2011), KIC 05952403 (= HD 181068; Derekas, Kiss \& Borkovits 2011), KICs 06543674, 07289157 (Slawson, Prša \& Welsh 2011), KIC 02856960 (Armstrong et al. 2012; Marsh, Armstrong \& Carter 2014), KIC 02835289 (Conroy et al. 2014), KICs 05255552, 06964043, 07668648 (Borkovits et al. 
2015), KIC 09007918 (Borkovits et al. 2016), and KIC 0415061 (= HD 181469), the latter of which is possibly at least a quintuple system (Shibahashi \& Kurtz 2012; Hełminiak et al. 2017). For 10 of these 11 CHTs, besides the outer eclipses, the inner binary also shows regular eclipses; hence, we call these CHTs 'triply eclipsing systems'. (The only exception is KIC 02835289 , where the inner binary is an ellipsodial light variable.) Though the much shorter duration of the $K 2$ observations is less favourable in regard to the discovery of systems with outer eclipses, another triply eclipsing CHT, HD 144548 was also identified in the C2 field of the extended $K 2$ mission (Alonso et al. 2015). Furthermore, recently, Hajdu, Borkovits \& Forgács-Dajka (2017) reported the discovery of two additional triply eclipsing CHTs, namely CoRoTs 104079133 and 221664856 amongst the EBs observed by the CoRoT space telescope (Auvergne et al. 2009).

Precise modeling of the brightness variations of these CHTs, especially during each outer eclipse, is a great challenge but, on the other hand, it offers huge benefits. This is so because the light curve is extremely sensitive to the complete configuration of the triple. As a consequence, even in the case where the outer orbit is wide enough to safely allow for the elimination of any dynamical perturbations in the analysis of accurate ETV and/or radial velocity (RV) curves, the same cannot be done for the light-curve solution. This is true because dynamically induced departures in the positions and velocities of the three bodies relative to a purely Keplerian motion, even if they are very small, will strongly affect all the characteristics of the forthcoming outer eclipses. Therefore, the accurate modeling of such systems, in most cases, requires a photodynamical approach, including the complete numerical integration of the motion of the three bodies, together with the simultaneous analysis of the light curve(s) (and, if it is available, the RV curve[s], as well), as was carried out by, e.g. Carter et al. (2011), for KOI-126 and Orosz (2015), for KIC $07668648 .^{2}$

In this paper we report the discovery and present the photodynamical analysis of the new triply eclipsing CHT EPIC 249432662 (= 2MASS J15334364-2236479, UCAC4 337-074729). The system was observed during Campaign 15 of the $K 2$ mission. Besides the regular, $\sim 3$ percent deep eclipses belonging to an 8.23 -d period, slightly eccentric EB, the Kepler spacecraft has observed an additional, 1.7-d-long, irregularly shaped fading event with an amplitude of almost 50 percent which we assumed to be an outer eclipse due to the presence of a third, distant, gravitationally bound stellar component. Ground-based spectroscopic and photometric follow-up observations have confirmed our assumption and made it possible to carry out a complete, joint photo-spectro-dynamical analysis of this 188.3-d-outer-period, triply eclipsing CHT, including the simultaneous joint analysis of $K 2$ and ground-based light curves, the ETV curve (derived from the photometric observations), and the ground-based RV curve, all accompanied by the numerical integration of the motion of the three bodies. We derive many of the parameters for this system. The paper is organized as follows. In Section 2 we describe the 80-d K2 observation of EPIC 249432662. Existing archival data on the target star are summarized in Section 3. Our two ground-based photometric follow-up campaigns, which led to the successful detection of further outer eclipses, are discussed

\footnotetext{
${ }^{2}$ In addition to KIC 07668648, J. Orosz has also successfully applied the same combined photodynamical approach to other CHTs amongst the Kepler-discovered EBs (e.g. to KIC 10319590) that did not exhibit outer eclipses, but the rapid eclipse depth variations and the features of the ETV and RV curves have allowed him to infer accurate system parameters.
}

in Section 4, while the ETV data determined from both the $K 2$ and ground-based photometry are briefly described in Section 5. In Section 6 we present our spectroscopic RV follow-up observations. We then use our improved photodynamical software package to model and evaluate the satellite and ground-based light curves, ETV curves, and RV results simultaneously (see Section 7). In Section 8 we discuss some of the astrophysical and orbital/dynamical implications of our solutions. We summarize our findings and draw some conclusions in Section 9. Finally, we discuss some practical details of our photodynamical code in Appendices A and B.

\section{K2 OBSERVATIONS}

Campaign 15 (C15) of the $K 2$ mission (Howell et al. 2014) was directed toward the constellation Scorpius between 2017 August 23 and 2017 November 20 for approximately 87 uninterrupted days. At the end of November, the $K 2$ Guest Observer (GO) Office made the $\mathrm{C} 15$ raw cadence pixel files (RCPF) publicly available on the Barbara A. Mikulski Archive for Space Telescopes (MAST). ${ }^{3}$ We utilized the RCPF in conjunction with the KADENZA software package $^{4}$ (Barentsen \& Cardoso 2018), in a manner similar to recent K2-discoveries (see e.g. Christiansen et al. 2108; David et al. 2018; Yu et al. 2018), combined with custom software in order to generate minimally corrected light curves. Due to limited processing power, some of us (MO, IT, HMS, MHK) generated a series of short-baseline $\mathrm{C} 15$ preview light curves, and carried out manual surveys with the lctools software (Kipping et al. 2015). The first C15 preview search identified EPIC 249432662 (proposed by GO15083, Coughlin) ${ }^{5}$ as a likely 8 -d eclipsing binary, while our second extended preview light curve identified an additional single, deep, compound eclipsing event of long duration centered at BJD $=2458018$.

Once the calibrated Ames data set was released, we downloaded all available $K 2$ extracted light curves common to Campaign 15 from the MAST. We utilized the pipelined data set of Vanderburg \& Johnson (2014) to construct the light curve, which is presented in Fig. 2; the top panel shows the data for all $80 \mathrm{~d}$ of the $K 2$ observation, whereas the bottom panel shows a 10-d zoom-in around the large eclipse that we dub the 'great eclipse' (or 'GE'). The two nearly equal-depth eclipses from the 8-d binary are also clearly evident. When looked at in the expanded view, the 'great eclipse' is seen to be composed of a deep and slightly asymmetric portion (here called 'GE1') and a sharp (i.e. short-duration) extra dip (called 'GE2') near the minimum of the event.

Note, however, that in our photodynamical analysis (see Section 7), we used a 'flattened' version of the light curve that differs from the one shown in Fig. 2 by having the long-term trend and low-frequency variability removed. The procedure is to iteratively fit a basis spline (B-spline) with breakpoints every $1.5 \mathrm{~d}$ to the light curve with the $3 \sigma$ outliers (including, of course, the eclipses) removed from the fit. This process is repeated until convergence is achieved (see Vanderburg \& Johnson 2014). The eclipses, including the great eclipse, are then added back to the spline fit.

We have examined both the $K 2$ pixel-level data and the PANStarrs image (Chambers et al. 2016) of the field to check on 'third-light' contamination to the $K 2$ light curve from neighbour stars. We utilized the pixel reference function of Kepler (on the module with the

\footnotetext{
${ }^{3}$ http://archive.stsci.edu/k2/data_search/search.php

${ }^{4}$ https://github.com/KeplerGO/kadenza

${ }^{5}$ https://keplerscience.arc.nasa.gov/data/k2-programs/GO15083.txt
} 



Figure 2. $K 2$ light curve of EPIC 249432662 from Campaign 15. Top panel: full 80-d light curve; bottom panel: 10-d zoom-in around the long, deep, and structured eclipse of the third star by the inner binary.

Great Eclipser; Bryson et al. 2010), which gives the contribution from a given star as a function of distance from the maximum in flux. The nearest star that could add contaminating light is $15^{\prime \prime}$ away and is only $\sim 20$ per cent the brightness of the target. The contribution from this star, according to the pixel reference function, is then no more than 0.1 per cent of the target star, and hence negligible.

Before doing any quantitative analysis, we qualitatively convinced ourselves that GE1 must be due to an eclipse of a third star in the system by one of the binary stars. This star would just happen to be moving in the same direction on the sky as the third star, and nearly at the same speed, so as to dramatically slow their relative motion and produce an eclipse that lasts for $1.7 \mathrm{~d}$. By contrast, GE2 must be caused by the other star in the binary that is moving in the opposite direction on the sky as the third star. We also concluded early on that the two stars in the binary must be of comparable mass, size, and $T_{\text {eff }}$, based on the near equality of the two binary eclipses. Finally, we realized that there was just a limited set of stellar parameters that would allow for only one star in the binary to be able to block $\sim 38$ per cent of the system light.

In the remainder of the paper we focus on understanding this 'great eclipse' quantitatively and, in the process, extracting the system parameters.

\section{ARCHIVAL DATA}

The target star image, as a composite of all three stars, has a $K e$ pler magnitude of 14.93. The coordinates of the target star and its brightness in other magnitude bands from the blue to the WISE 3 band are summarized in Table 1 . The new Gaia DR2 release puts
Table 1. Photometric properties of EPIC 249432662.

\begin{tabular}{lc}
\hline Parameter & EPIC 249432662 \\
\hline Aliases & 2MASS J15334364-2236479 \\
& WISE J153343.62-223648.1 \\
& UCAC4 337-074729 \\
& Gaia DR2 6239702584685025280 \\
RA (J2000) & $15: 33: 43.639$ \\
Dec. $(\mathrm{J} 2000)$ & $-22: 36: 48.07$ \\
$K_{p}$ & 14.93 \\
$B$ & $16.57^{a}$ \\
$V$ & $15.46^{a}$ \\
$G$ & $14.92^{b}$ \\
$r^{\prime}$ & $14.95^{a}$ \\
$i^{\prime}$ & $14.45^{a}$ \\
$\mathrm{~J}$ & $12.83^{c}$ \\
$\mathrm{H}$ & $12.25^{c}$ \\
$\mathrm{~K}$ & $12.09^{c}$ \\
$\mathrm{~W} 1$ & $11.99^{d}$ \\
$\mathrm{~W} 2$ & $11.96^{d}$ \\
$\mathrm{~W} 3$ & $11.71^{d}$ \\
Distance $(\mathrm{pc})$ & $445 \pm 7^{b}$ \\
$\mu_{\alpha}\left(\mathrm{mas} \mathrm{yr}^{-1}\right)$ & $-18.70 \pm 0.07^{b}$ \\
$\mu_{\delta}\left(\mathrm{mas} \mathrm{yr}^{-1}\right)$ & $-10.82 \pm 0.05^{b}$
\end{tabular}

Notes. ${ }^{\mathrm{a}}$ UCAC4 (Zacharias et al. 2013). ${ }^{\mathrm{b}}$ Gaia DR2 (Lindegren et al. 2018). ${ }^{c}$ 2MASS archive (Skrutskie et al. 2006). ${ }^{\mathrm{d}}$ WISE archive (Cutri et al. 2013).

the target at a distance of $445 \pm 7 \mathrm{pc}$. This distance and the corresponding proper motions from Gaia DR2 are also listed in Table 1. Note, however, that despite the unprecedented astrometric precision of Gaia, the DR2 parallax, and therefore distance, for the present system should be considered as only a preliminary value, and should not be accepted without some caveats. The reason is that binary star solutions have not yet been incorporated into the DR2 results. In particular, since the orbital period of the outer orbit in our triple is $P_{2} \sim 188 \mathrm{~d}$, which is almost exactly half of the orbital period of the Gaia satellite, the absence of corrections for the internal motions might be critical for our triple.

Based on the Gaia photometry and the source distance, the DR2 file on this object lists the star as having a radius of $0.86_{-0.077}^{+0.055} R_{\odot}$. Of course, this analysis is based on the assumption that there is one dominant star present, and the light from the two stars in the 8-d binary do not contribute much to the system light.

Under these assumptions we know at least that the third star is of $\mathrm{K}$ spectral type with a mass of $\sim 0.7-0.8 M_{\odot}$, and lies quite close to the zero-age main sequence.

\section{GROUND-BASED FOLLOW-UP OBSERVATIONS}

The history of our ground-based follow-up observations nicely illustrates the role of good fortune in a scientific endeavour. Our preliminary joint photodynamical runs, including the $K 2$ photometry and the ETV curves derived from it, clearly demonstrated that the main features of the $K 2$ light curve can be well reproduced with a compact, dynamically active, triple stellar system in an almost coplanar configuration. However, due to the strong degeneracies among many of the orbital parameters in terms of the outer eclipsing pattern, we were unable to constrain the outer orbital period and, therefore, to predict the likely time(s) of the forthcoming outer eclipses. This situation changed dramatically after we obtained the fourth RV data point from 2018 March 22. This RV point made it possible to constrain the outer period with only an $\sim 2-3$-d un- 
certainty and, therefore, to predict the most probable forthcoming outer eclipse times to within a range of only a few days. The most important consequence was that we then understood that the forthcoming outer eclipses should be occurring within 5-10 d of that time! Therefore, we had to urgently organize an international observing campaign with several observers from Arizona to Chile. We were thereby able to perfectly catch the next pair of 'great (outer) eclipses' almost exactly after the start of the follow-up ground-based observations.

\section{JBO observations}

The observations of the primary third-body eclipses were conducted with the Junk Bond Observatory by the author TGK. The telescope is an 80-cm Ritchie Chretien with an SBIG STL6303E CCD. 60-s unfiltered images were shot sequentially through the events. Darks, flats, and data images were reduced using the MAXIMDL software by Bruce Gary.

The observations were carried out for 5 and $6 \mathrm{~h}$ on the evenings of 2018 March 27 and 29, respectively. By good fortune, both of the deep primary third-body eclipses were captured photometrically.

\section{RoBoTT telescope observations}

We have carried out photometry of the target star at the end of 2018 March with the ROBOTT telescope (formerly VYSOS6). The images are taken in two filters: sloan $r^{\prime}$ and $i^{\prime}$, during the first night with exposure times of $30 \mathrm{~s}$, and then later at $60 \mathrm{~s}$. Typically nine images are combined with outlier rejection to remove cosmic rays. The images are taken at the same sky position, and the source of interest is at the image center. While the FoV of the instrument is $2.7 \times 2.7 \mathrm{deg}$, we extracted submaps of $45 \operatorname{arcmin} \times 45 \mathrm{arcmin}$ FoV centred on the target, and used only stars in this area for the light curve processing. A description of the data processing and reduction can be found in Haas, Hackstein \& Ramolla (2012).

\section{DEMONEXT observations}

We obtained additional ground-based photometry for EPIC 249432662 using the DEMONEXT telescope (Villanueva et al. 2018) at Winer Observatory in Sonoita, Arizona. DEMONEXT is a $0.5 \mathrm{~m}$ PlaneWave CDK20 telescope with a $2048 \times 2048$ pixel FLI Proline CCD3041 camera, a $30.7 \operatorname{arcsec} \times$ 30.7 arcsec field of view, and a pixel scale of $0.9 \mathrm{arcsec}_{\mathrm{pixel}}{ }^{-1}$.

EPIC 249432662 was placed in the DEMONEXT automated queue for continuous monitoring for the night of 2018 March 28. 250 observations were executed while the target was above airmass 2.4. An exposure time of $42 \mathrm{~s}$ was used and DEMONEXT was defocused to avoid saturation.

All observations were made with a sloan- $i^{\prime}$ filter, and were reduced using standard bias, dark, and flat-fielding techniques. Relative aperture photometry was performed using AIJ (Collins et al. 2017) on the defocused images to obtain the time-series light curve. No detrending parameters were used in the initial reductions.

During these observations, the next regular primary eclipse of the 8-d binary, occurring shortly after the second great eclipsing event, was successfully observed.

We also organized a second follow-up ground-based observing campaign about 3 months later to catch the secondary outer eclipses (i.e. the events when the members of the 8-d binary were eclipsed by star $\mathrm{A}$ in its outer orbit). We were less fortunate in observing these events than before; however, partial observations of one of the two predicted events, as well as some additional, away-from-outereclipse observations made it possible to further narrow the error bars on some of the orbital parameters. Furthermore, during this campaign, an additional secondary eclipse of the 8-d binary was also observed. The following observatories took part in this second campaign.

\section{PEST observations}

The end of the egress phase of a secondary outer eclipse event (i.e. when one of the stars in the 8-d binary emerges from behind the disc of star A) during 2018 July 9 was observed in the $R_{\mathrm{C}}$ band at PEST observatory, which is a home observatory with a 12-inch Meade LX200 SCT f/10 telescope with an SBIG ST-8XME CCD camera. The observatory is owned and operated by Thiam-Guan (TG) Tan. PEST is equipped with a BVRI filter wheel, a focal reducer yielding f/5, and an Optec TCF-Si focuser controlled by the observatory computer. PEST has a 31 arcmin $\times 21$ arcmin field of view and a 1.2 arcsec per pixel scale. PEST is located in a suburb of the city of Perth, Western Australia. The target was also observed during the next two consecutive nights. On the night of 2018 July 10 , no systematic light variations were observed, while the last observation on 2018 July 11 caught a regular secondary eclipse of the 8-d binary. These two observations, however, were not included in our analysis, since the same 8-d binary eclipse was also observed with a larger aperture telescope, which naturally produced a light curve with significantly less scatter (see below).

\section{LCO observations}

Data with the Las Cumbres Observatory (LCO; Brown et al. 2013) were obtained from 2018 July 9 to 15 . LCO is a fully robotic network of telescopes, deployed around the globe in both hemispheres. ${ }^{6}$ Observing requests are entered online, including the required telescope aperture and other technical information (e.g. exposure time and band), and the scheduling software decides in which site to carry out the observation and with which telescope (many of the LCO sites contain 2-3 telescopes of the same aperture). All data obtained by LCO telescopes are reduced by an automated pipeline and made available to the users. We have carried out the photometric analysis of all LCO data obtained here using the AstroImageJ pipeline (Collins et al. 2017).

Data with an LCO $0.4 \mathrm{~m}$ telescope in Siding Spring, Australia, were obtained on the local night of 2018 July 15 . LCO $0.4 \mathrm{~m}$ telescopes are mounted with an SBIG camera, and this data set was obtained with an exposure time of $150 \mathrm{~s}$ and the Pan-STARRS-w filter, while applying a telescope defocus to avoid saturation. The 2018 July 15 data set includes an ingress to a regular 8-d binary primary eclipse. However, due to its higher photometric scatter and the missing egress phase, this resulted in an outlier ETV value and, therefore, it was omitted from the analysis.

Data with LCO $1.0 \mathrm{~m}$ telescopes were obtained at CTIO, Chile, on the local nights of 2018 July 8 and 10, and at SAAO, South Africa, on the local nights of 2018 July 9 and 12 . LCO $1.0 \mathrm{~m}$ telescopes are mounted with a SINISTRO camera, where we used the SDSS $-i^{\prime}$ band and an exposure time of $250 \mathrm{~s}$, while defocusing the telescope by 1.0. The 2018 July 8 data includes an ingress to the first secondary outer eclipse, while the other 3 data sets obtained with the $1.0 \mathrm{~m}$ telescopes show a flat light curve.

Data with the LCO $2.0 \mathrm{~m}$ Faulkes Telescope South (FTS) at Siding Spring, Australia, were obtained on the local nights of 2018 July 9 and 11. FTS is mounted with a Spectral camera, where we used the SDSS- $i^{\prime}$ band, an exposure time of $70 \mathrm{~s}$, and defocused the telescope by 1.0. The July 11 data show most of the same regular secondary eclipse of the 8-d binary, which was also observed at

\footnotetext{
${ }^{6}$ For updated information about the network see: https://lco.global
} 
PEST Observatory (see above), and the July 9 data show a flat light curve.

For the combined photodynamical analysis (see Section 7) the data points of each night, given in magnitudes, were converted into linear fluxes, averaged into bins with a cadence time of $\sim 15 \mathrm{~min}$, and then normalized so that the out-of-eclipse level for each night is close to unity. Then those individual data sets, which were selected for further analysis, were subdivided into two groups, according to the different filters used in the data collect. The sloan $i^{\prime}$ light curves of the DEMONEXT and LCO 1.0 and $2.0 \mathrm{~m}$ observations were collected into one file, while the JBO observations and the July 9 PEST observations were added to the second light-curve file that was designated as $R_{C}$-band observations.

The most problematic aspect of forming these light curves was in finding the correct, common, out-of-eclipse flux level for the different observations. This was especially problematic for those observations where relatively little out-of-eclipse data was recorded. In order to refine our initial estimated normalizations, after some preliminary photodynamical runs (see Section 7), we renormalized each night's light-curve segment via the use of our synthetic model light curves. In summary, we cannot exclude the possibility of some minor systematic effects due the inexact light-curve normalizations. However, we expect that these systematic errors due to the uncertain out-of-eclipse-levels should be smaller than the random errors from the statistical scatter of the individual data points even after the 15-min time binning.

\section{ETV DATA}

As usual, our first step in confirming our hierarchical triple-star hypothesis was to check whether the regular eclipses of the 8-d eclipsing binary does indeed exhibit ETVs. Therefore, we determined the mid-eclipse times of the shallow binary eclipses, and generated ETV curves. The method was the same one we used in several of our previous works, and is described in detail in Borkovits et al. (2016). For the $\sim 80$-d-long $K 2$ observation we were able to determine the eclipse times of 10 primary and 11 secondary eclipses, which include all but one of the eclipsing events during Campaign 15. The only missing event, a primary eclipse at BJD 2458018 , occurred during the great eclipse and, therefore, it cannot be distinguished from the composite light curve. Later, during the two ground-based follow-up campaigns, three additional 8-d binary eclipses were also observed. The mid-eclipse times of these events were also calculated in the same manner as for the eclipses observed with $K 2$. Note, however, that for the very last event, due to insufficient coverage and larger photometric scatter, an outlier ETV result was obtained; therefore, this point was excluded from the subsequent analysis. All the mid-eclipse times that we obtained are tabulated in Table 2. The resultant ETV curves clearly reveal highly significant variations due to physical interactions among the stars. The combined photodynamical analysis of these ETV curves, together with the light curves and RV data, is described later in Section 7.

\section{RV DATA}

\section{Keck HIRES observations}

Using the Keck I telescope and HIRES instrument, we collected four observations of EPIC 249432662 from 2018 February 1 to 2018 May 25 UT using the standard California Planet Search setup (Howard et al. 2010). With a visual magnitude of 14.9, the C2 decker $(0.87$ arcsec $\times 14.0$ arcsec was required for sky subtraction, allowing the removal of night sky emission lines and scattered moonlight that can inhibit the determination of systemic radial velocities and stellar properties. With a resulting spectral resolution of $\sim 60000$, each of the four 10-min exposures resulted in an SNR $\sim 15$ at $5000 \AA$. We searched each of the four spectra for the presence of secondary spectral features due to the companion M-dwarfs and found no evidence of the companions down to a level of 1 per cent of the flux of the primary, and outside the separation of $\pm 10 \mathrm{~km}$ $\mathrm{s}^{-1}$ (Kolbl et al. 2015). This is consistent with the expected flux from the two M-dwarf companions. Knowing that the primary star dominates the flux of the system, we calculated the systemic RV of the system using the telluric absorption lines in the HIRES red chip (Chubak et al. 2012) resulting in values with uncertainties of $0.2 \mathrm{~km} \mathrm{~s}^{-1}$ (Table 3).

\section{McDonald Tull spectrograph observations}

We observed EPIC 249432662 on three different occasions with the high-resolution Tull spectrograph (Tull et al. 1995) on the 2.7-m telescope at McDonald Observatory in Ft. Davis, TX. We observed using a 1.2-arcsec wide slit, yielding a resolving power of 60000 over the optical band. On each night, we obtained 3-4 individual spectra back to back with 20-min exposures to aid in cosmic-ray rejection, which we combined in post-processing to yield a single higher signal-to-noise spectrum. We bracketed each set of exposures with a calibration exposure of a ThAr arc lamp to precisely determine the spectrograph's wavelength solution. We extracted the spectra from the raw images and determined wavelength solutions, using standard IRAF routines, and we measured the star's absolute radial velocity using the KEA software package (Endl \& Cochran 2016).

The seven radial velocities we were able to obtain for that outerorbit star are shown in Fig. 3. The observation times and RVs with uncertainties are given quantitatively in Table 3 . There is marginally sufficient information to fit these seven points to a general eccentric orbit, and we do not report that attempt here. Instead, we fit these RV points simultaneously with all the other photometrically obtained data, using our photodynamics code (see Section 7).

We determined the stellar properties of this primary star using each of the four HIRES spectra using the SpecMatch-emp routine (Yee, Petigura \& von Braun 2017). The observed spectra are each shifted to the observatory rest frame, de-blazed and compared, in a $\chi^{2}$-squared sense to a library of previously observed HIRES spectra that span the main sequence. The best matches are determined and the values are a weighted average of the mostly closely matching spectra. The average stellar properties resulting from analyzing all four spectra are $T_{\text {eff }}=4672 \pm 100 \mathrm{~K}, R_{\text {star }}=0.77 \pm 0.1 R_{\odot}$, and metallicity $=0.09 \pm 0.1$. The results are given in Table 3 .

\section{COMBINED PHOTODYNAMICAL ANALYSIS}

The compactness (i.e. the small $P_{2} / P_{1}$ ratio) of this hierarchical triple indicates that the orbital motion of the three stars is expected to be significantly non-Keplerian, even over the time-scale of the presently available data. Hence, an accurate modeling of the observed photometric and spectroscopic data, and therefore a proper determination of the system parameters, requires a complex photodynamical analysis. This consists of a combined joint analysis of the light curve, ETV curve, and RV curve with a simultaneous numerical integration of the orbital motion of the three stars.

The analysis was carried out with our own software package LIGHTCURVEFACTORY (Borkovits et al. 2013; Rappaport et al. 2017; Borkovits et al. 2018). This code is able to emulate simultaneously the photometric light curve(s) of triply eclipsing triple stars (in different filter bands), the RV curves of the components (including 
Table 2. Mid-times of primary and secondary eclipses of the inner pair EPIC 249432662Bab.

\begin{tabular}{lcccccccc}
\hline $\begin{array}{l}\text { BJD } \\
-2400000\end{array}$ & $\begin{array}{c}\text { Cycle } \\
\text { no. }\end{array}$ & $\begin{array}{c}\text { SD } \\
(d)\end{array}$ & $\begin{array}{c}\text { BJD } \\
-2400000\end{array}$ & $\begin{array}{c}\text { Cycle } \\
\text { no. }\end{array}$ & $\begin{array}{c}\text { SD } \\
(d)\end{array}$ & $\begin{array}{c}\text { BJD } \\
-2400000\end{array}$ & $\begin{array}{c}\text { Cycle } \\
\text { no. }\end{array}$ & $\begin{array}{c}\text { SD } \\
(d)\end{array}$ \\
\hline 57989.55756 & -0.5 & 0.00304 & 58026.67394 & 4.0 & 0.00089 & 58059.64242 & 8.0 & 0.00091 \\
57993.68484 & 0.0 & 0.00490 & 58030.79369 & 4.5 & 0.00335 & 58063.76072 & 8.5 & 0.00312 \\
57997.80082 & 0.5 & 0.00059 & 58034.92167 & 5.0 & 0.00329 & 58067.88414 & 9.0 & 0.01844 \\
58001.92930 & 1.0 & 0.00597 & 58039.04110 & 5.5 & 0.00098 & 58071.99798 & 9.5 & 0.00516 \\
58006.04589 & 1.5 & 0.00208 & 58043.16586 & 6.0 & 0.00234 & 58076.12270 & 10.0 & 0.00240 \\
58010.17639 & 2.0 & 0.00237 & 58047.28020 & 6.5 & 0.00350 & 58207.96333 & 26.0 & 0.00113 \\
58014.29779 & 2.5 & 0.00216 & 58051.40438 & 7.0 & 0.00361 & 58310.96132 & 38.5 & 0.00042 \\
58022.54777 & 3.5 & 0.00106 & 58055.52069 & 7.5 & 0.01377 & 58315.06936 & 39.0 & 0.00254 \\
\hline
\end{tabular}

Notes. Integer and half-integer cycle numbers refer to primary and secondary eclipses, respectively. Most of the eclipses (cycle nos. -0.5 to 10.0$)$ were observed by Kepler spacecraft. The last three eclipses were observed by ground-based telescopes, and the very last point was omitted from the analysis.

Table 3. Radial velocity study.

EPIC 249432662

RV measurements:

BJD-2400000

$58151.1240^{a}$

$58161.1477^{a}$

$58173.9962^{b}$

$58200.1271^{a}$

$58261.8494^{b}$

$58263.8553^{a}$

$58291.7400^{b}$

Spectroscopic parameters:

$T_{\text {eff }}[\mathrm{K}]$

$R\left[\mathrm{R}_{\odot}\right]$

$\mathrm{Fe} / \mathrm{H}[\mathrm{dex}]$

Notes. ${ }^{\mathrm{a}}$ Keck HIRES data; ${ }^{\mathrm{b}} \mathrm{McD}$ onald data.

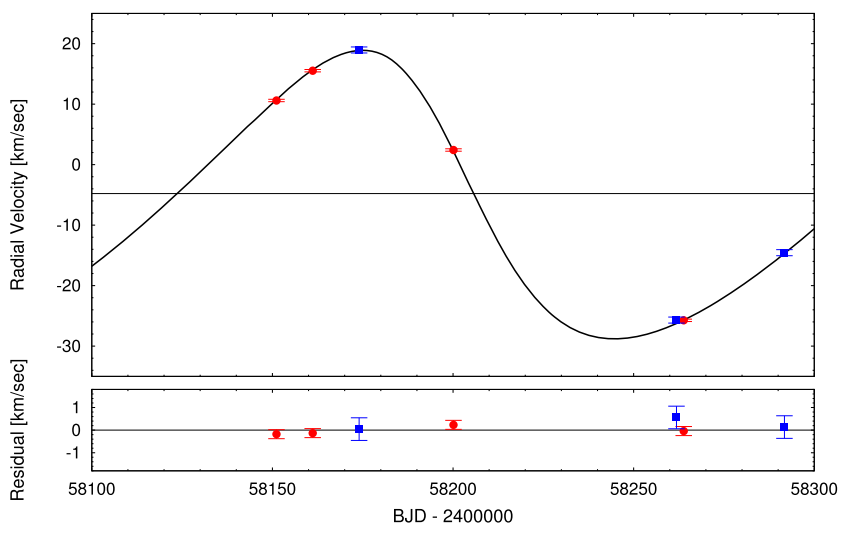

Figure 3. Radial velocity measurements of the brightest, outer component of EPIC 249432662 together with the photodynamical model RV curve (top panel) and the residuals (bottom). Red circles and blue squares denote Keck HIRES and McDonald points, respectively. The thin horizontal line in the upper panel shows the RV value at the conjunction points (i.e. when the sum of the true anomaly and the argument of periastron of the outer orbit is equal to $\pm 90^{\circ}$ ). See Section 7 for a description of the photodynamical model in which the RV points were included in the fit.

modeling of higher order distortions of the RV curves due to e.g. Rossiter-McLaughlin effect and ellipsoidal light variations), and the ETV curves (both primary and secondary) of the inner binary. Furthermore, the motions of the three bodies, optionally, can be in- tegrated numerically (as in the present case) or can be treated as the sum of two Keplerian motions (as is usual for hierarchical triples with negligible short-term dynamical perturbations). The built-in numerical integrator is a seventh-order Runge-Kutta-Nyström integrator (Fehlberg 1974), and is identical to that which was described in Borkovits et al. (2004). (In Appendix A we also discuss some of the practical issues regarding the use of a numerical integrator in photodynamical modeling.) Furthermore, independent of whether unperturbed Keplerian motion or numerical integration is applied, the software takes into account the 'LTTE' by computing the apparent positions of the stars when light from each of them actually arrives at the Earth. Therefore, the LTTE is inherently built into the model light curves.

The LIGHTCURVEFACTORY code also employs a Markov Chain Monte Carlo (MCMC)-based parameter search, using our own implementation of the generic Metropolis-Hastings algorithm (see e.g. Ford 2005). Apart from the inclusion of the ETV curves, and the numerical integration of the orbital motion, the basic approach and steps of this study are similar to those which were followed during the previous non-photodynamical analyses of two doubly eclipsing quadruple systems EPIC 220204960 (Rappaport et al. 2017, Section 7) and EPIC 219217635 (Borkovits et al. 2018, Section 7).

Here we concentrate mainly on the differences in the LIGHTCURVEFACTORY code used in this work compared to the previous studies mentioned above. The most noteworthy new feature about the present system is the existence of outer eclipses, i.e. when the inner binary occults the third star in the system or, vice versa, when the third star eclipses one or both members of the inner binary. This carries significant extra information about the geometrical configuration of the entire triple system, including both astrophysical and key orbital parameters. For example, as was shown in a number of previous studies (see e.g. Carter et al. 2011; Borkovits et al. 2013; Masuda et al. 2015), the precise brightness variations during outer eclipses, including the timings, durations, depths, and fine structure of the eclipses, depend extraordinarily strongly on the physical dimensions of the system and, therefore, on the masses of the components. Similarly, the third-body eclipse structure depends very strongly on the orientations of the two orbits, both relative to each other and to the observer.

Furthermore, given the compactness of this triple, the $P_{2}$-timescale dynamical perturbations not only strongly influence the properties of the outer eclipsing events, but also dominate the ETVs of the regular eclipses of the inner EB. This fact also offers the very good possibility of obtaining accurate orbital and dynamical parameters for this triple (see Borkovits et al. 2015, for a detailed theoretical background.). 
As a consequence, during our MCMC parameter search we typically jointly fit the following five data series ${ }^{7}$ :

(i) The processed, 'flattened' $K 2$ light curve;

(ii) Two sets of $\left(R_{\mathrm{C}^{-}}\right.$and $i^{\prime}$-band) ground-based photometric observations;

(iii) The RV curve of the brightest, outer component; and

(iv) The ETV curves of the inner EB (for both primary and secondary eclipses).

Some of these items require some further explanation. Starting with item (i), we used two different versions of the $K 2$ light curve. We made a series of MCMC runs using the complete flattened $K 2$ light curve (hereafter 'complete' light curve), and another series where the out-of-eclipse sections of the light curve were eliminated from the fit (hereafter 'eclipses-only' light curve). The latter results in a data train that consists only of the great eclipse itself plus a narrow window of width $\sim 0.33 \mathrm{~d}$ centered on each 8 -d-eclipse. Dropping the out-of-eclipse sections can be easily justified by noting the spherical shape of the stars and, consequently, the lack of any measurable ellipsoidal variations (ELVs). (Note that the lack of ELVs as well as any irradiaton effect were already invoked to justify flattening the light curve.) There are at least two advantages in omitting the out-of-eclipse light-curve sections. As noted, in the present long-cadence $K 2$ light curve each 8-d-binary eclipsing event contains only 5-6 data points, as opposed to hundreds of points in the out-of-eclipse sections. Therefore, dropping out these latter points makes the $\chi^{2}$-probe more sensitive to the light-curve features during the 8-d binary eclipses and, it also saves much of the computational time.

When we fit the 'complete' $K 2$ light curve, we did not employ any correction for the long-cadence time because of the high computational costs. However, for the fits to the 'eclipses-only' $K 2$ light curve we corrected the model light curve for the $\sim 29$.4-min longcadence time of Kepler. ${ }^{8}$ We find that our fits to the 'complete' light curve and 'eclipses-only' light curve result in very similar parameter values. The differences are far below the $1 \sigma$ uncertainties for most of the fitted and computed parameters except for the inclination angle of the 8-d binary $\left(i_{1}\right)$. For $i_{1}$, not surprisingly, we found a bit higher values (by $\sim 0.1-0.2$ ) in the cadence-corrected runs. Therefore, in the forthcoming discussion we refer to the results obtained from the 'eclipses-only' runs.

Turning to item (iv), i.e. the RV curve, a visual inspection leads us to believe that there might be a few hundred $\mathrm{m} \mathrm{s}^{-1}$ offset between the Keck HIRES and the McDonald measurements. One way to handle such discrepancies in the case of multisite spectroscopic data is to introduce an 'offset' term for each instrument as additional parameters to be fitted. In our case, however, we have only seven RV points (and two of them were taken almost at the same epoch), but there are seven parameters required for the complete solution to a purely Keplerian RV orbit (amplitude, $K$, period, $P$, eccentricity, $e$, argument of periastron, $\omega$, periastron passage, $\tau$, or their equivalents, systemic velocity, $\gamma$, and the RV offset between the two instruments). Thus, at least in terms of an analysis of the RV curve by itself, we would encounter the problem of zero degrees of freedom. Thus, instead of introducing an additional offset parameter, we constrained only a single systemic velocity $(\gamma)$ parameter

\footnotetext{
${ }^{7}$ In some circumstances we jointly fit only a subset of these time series.

${ }^{8}$ In the case of the cadence-time correction our code for each data point calculate five flux values evenly spaced within the cadence time, and then computes a net flux using Simpson's rule.
}

(see below). However we checked the effect of a potential RV offset a posteriori. This was done as follows. After obtaining a tentative solution with the joint photodynamical analysis, we calculated the averages of the RV residuals for the two sources of the RV data, and accepted their difference as a probable offset. Then we subtracted this value $\left(\Delta \gamma=217 \mathrm{~m} \mathrm{~s}^{-1}\right)$ from the McDonald points and made an additional joint photodynamical MCMC run, with the original RV curve replaced with this slightly modified one. We found from this exercise that the effect of any RV offset remains far below the $1 \sigma$ parameter uncertainties. In particular, we found that the stellar masses differed by less than 1 per cent in either analysis. Therefore, we conclude that the presence of any small, but uncertain, RV offset has no influence on the accuracy of our parameter determination.

Regarding item (v) above, one may make the counter-argument that the accurate timings of the inner, regular eclipses are already inherent in the light-curve analysis and, therefore, the inclusion of the ETV curves into the fitting process would be unnecessarily redundant. While, in theory, this is evidently true, we decided to use the ETV curves for two practical reasons. First, this treatment allows us to give much higher weight to that part of the timing data that carries crucial information about the dynamics of such triple systems. By contrast, as was mentioned above, the full $K 2$ light curve contains only 5-6 data points in each 8 -d binary eclipsing event, compared to hundreds of points in the out-of-eclipse region. This fact makes it almost impossible to fine-tune the timing data with a $\chi^{2}$-probe of the 'complete' light-curve fit. By contrast, the ETV curve, which is an extract of all the timing data, and contains most of the dynamical information, becomes very sensitive to even the smallest changes in the key parameters (not just the binary period). Interestingly, we found that the same argument also remains valid for the 'eclipses-only' $K 2$ light-curve runs. In our opinion, this is so because the nearly 2 -d-long great eclipse itself contains nearly the same number of data points as all of the brief 8-d-binary eclipsing events combined. This results in an overoptimization of the great eclipse at the expense of the 8-d binary eclipses. Secondly, the inclusion of the ETV curve into the photodynamical analysis has allowed us to constrain the inner orbital period during each trial step, as will be discussed below in Appendix A. The practical way in which we produced the numerical model ETV data is also described in Appendix B.

During our analysis we carried out almost a hundred MCMC runs, and tried several sets and combinations of adjustable parameters. We also applied a number of physical (or technical) relations to constrain some of the parameters in order to reduce the degrees of freedom in our problem. For example, in some preliminary runs we tried to constrain the masses and/or the radii of some of the stars via empirical mass-radius-temperature relations available for main-sequence stars (e.g. Tout et al. 1996; Rappaport et al. 2017; Appendix), but these runs resulted in significantly higher $\chi^{2}$ values and, therefore, we stopped applying such constraints. By contrast, we found it worthwhile to apply some technical (or, mathematical) constraints to the systemic radial velocity $\gamma$, the period $P_{1}$, and the reference primary eclipse time $\left(T_{0}\right)_{1}$ of the inner binary. In the case of $\gamma$, which is practically independent of any other parameter, its best-fitting value was calculated a posteriori in each trial run with a linear regression by minimizing $\chi_{\mathrm{RV}}^{2}$ of the actual model RV curve. In the case of $P_{1}$ and $\left(T_{0}\right)_{1}$ the ETV model was used for the constraining process, and it is idealized and described in Appendix A.

For the final runs, we ended up adjusting 18 parameters, as follows: 
(i) Three parameters related to the remaining orbital elements of the inner binary: eccentricity $\left(e_{1}\right)$, the phase of the secondary eclipse relative to the primary one $\left(\phi_{\mathrm{sec}, 1}\right)$ that constrains the argument of periastron $\left(\omega_{1}\right)$, and inclination $\left(i_{1}\right)^{9}$;

(ii) Six parameters related to the outer orbital elements: $P_{2}$, $e_{2} \sin \omega_{2}, e_{2} \cos \omega_{2}, i_{2}$, the time of the superior conjunction of the tertiary outer star $\left(T_{0}\right)_{2}$, and the longitude of the node of the outer orbit of the tertiary $\left(\Omega_{2}\right)^{10}$;

(iii) Three mass-related parameters: the spectroscopic mass function of the outer orbit $f_{2}\left(m_{\mathrm{B}}\right)$, the mass ratio of the inner orbit $q_{1}$, and the mass of the tertiary, which is the most massive component $m_{\mathrm{A}}$;

(iv) and, finally, six other parameters that are related (almost) exclusively to the light-curve solutions, as follows: the fractional radii of the inner binary stars $R_{\mathrm{Ba}} / a_{1}, R_{\mathrm{Bb}} / a_{1}$, the physical radius of the tertiary $R_{\mathrm{A}}$, the temperature of the tertiary $T_{\mathrm{A}}$, the temperature ratio of the primary of the inner binary and the tertiary $T_{\mathrm{Ba}} / T_{\mathrm{A}}$ and, the temperature ratio of the two components of the inner binary $T_{\mathrm{Bb}} / T_{\mathrm{Ba}}$.

The adjustment of $T_{\mathrm{A}}$ warrants some further explanation. This parameter is a natural output of the spectroscopic analysis (see Section 6), but it has only a minor influence on the light curve through the different relative eclipse depths in the three photometric bands. Therefore, our original idea was to take the results of our spectroscopic analysis and then use Gaussian priors for this parameter to obtain the effective temperature of the tertiary from the complex analysis. However, we found that this was too constraining on $T_{\mathrm{A}}$, and in particular led to model $\mathrm{V}$ magnitudes that were too high and inferred distances to the source that were significantly closer than that given by Gaia. Thus, we ultimately replaced the Gaussian prior on $T_{\mathrm{A}}$ with a uniform prior that was centered on the spectroscopic result, but the boundaries of the allowed parameter domain were expanded to somewhat beyond the $2 \sigma$ uncertainties of the spectroscopic results.

Regarding the other parameters, similar to our approach with two quadruple systems (Rappaport et al. 2017; Borkovits et al. 2018), we applied a logarithmic limb-darkening law, where the coefficients were interpolated from the pre-computed passbanddependent tables in the PHOEBE software (Prša \& Zwitter 2005). The PHOEBE-based tables, in turn, were derived from the stellar atmospheric models of Castelli \& Kurucz (2004). Considering the gravity darkening exponents, for the nearly spherical stellar shapes in our triple, their numerical values have only a negligible influence on the light-curve solution. Thus, instead of using the recent results of Claret \& Bloemen (2011) that tabulate stellar parameters and photometric system-dependent gravity-darkening coefficients in a three-dimensional grid, and would therefore require some further interpolation, we simply adopted a fixed value of $g=0.32$. This is appropriate for late-type stars according to the traditional model of Lucy (1967). We also found that the illumination/reradiation effect was quite negligible for all three stars; therefore, in order to save computing time, this effect was not taken into account. As a

\footnotetext{
${ }^{9}$ For the rigorous meaning of the orbital elements in a photodynamical problem, see Appendix A.

${ }^{10}$ The dynamical perturbations are sensitive only to the difference in the nodes $\left(\Delta \Omega=\Omega_{2}-\Omega_{1}\right)$. Hence we set $\Omega_{1}=0^{\circ}$, and it was not adjusted during the runs. In such a manner, adjusting $\Omega_{2}$ is practically equivalent to the adjustment of $\Delta \Omega$. On the other hand, however, note that due to the third-body perturbations $\Omega_{1}$ was also subject to low-amplitude variations during each integration run.
}

consequence of using a flattened $K 2$ light curve (see Section 2), which was assumed to be flat during the out-of-eclipse regions, we decided that in contrast to our previous work, we would not take into account the Doppler-boosting effect (Loeb \& Gaudi 2003; van Kerkwijk et al. 2010).

Furthermore, in the absence of any information on the rotation properties of any of the stars, we assumed that the inner binary members rotate quasi-synchronously with their orbit. ${ }^{11}$ For simplicity, for the third, outer stellar component we supposed that its rotational equatorial plane is aligned with the plane of the outer orbit, while its spin angular velocity was arbitrarily set to be 10 times larger than its orbital angular velocity at periastron (which resulted in an $\sim 11.5$-d rotational period). These arbitrary choices, however, have no significant influence on the light-curve solution.

Finally, we note that due to the absence of any significant additional contaminating light in the $K 2$ aperture (see Section 2) we set the extra light parameter consistently to $\ell_{\mathrm{x}}=0$ for all the three light curves (i.e. no light contamination was considered).

The orbital and astrophysical parameters obtained from the joint photodynamical analyses are given in Table 4 . The corresponding model light curves for different sections of the observed lighcurves are presented in Figs 4, 5, and 6, while the RV-curve portion of the solution was shown previously in Fig. 3. Finally, the model ETV curve plotted against the observed ETVs is shown in Fig. 7.

\section{DISCUSSION OF THE RESULTS}

\subsection{Astrophysical properties}

Our photodynamical analysis of the available data for EPIC 249432662, the 'great eclipser', has led to a reasonably wellconstrained set of system parameters (see Table 4). Among these are the masses of the three constituent stars, which are determined well enough to make a contribution to the collection of empirically well-measured radii and masses of stars on the lower main sequence. We plot the $R(M)$ points for the three stars in the 'great eclipser', with error bars in Fig. 8. Also shown in the figure are two sets of theoretical stellar models, as well as a number of wellmeasured stars in binary systems. We can see that the three great eclipser stars lie somewhat above the stellar model locations, but comfortably in among the collection of other well-measured stars in binary systems. The usual explanation for the somewhat larger radii of the measured systems is thermal 'inflation' due to interactions in the binary system, e.g. tidal heating (see e.g. Han et al. 2017, and references therein).

A fundamental check on the system parameters that we have found can be made by computing the photometric parallax for the target, and then comparing it to the Gaia distance. To obtain this quantity using our photodynamically determined stellar radii and temperatures, we calculate the bolometric luminosities and, thereby, the absolute bolometric magnitude of each star. Then, these values are converted into absolute visual magnitudes via the formulae of Flower (1996) and Torres (2010). Finally we compute the net absolute visual magnitude for the system as a whole, and obtain $M_{V}=$ $6.57 \pm 0.14$. We then utilized a web-based applet ${ }^{12}$ to estimate $E(B$

\footnotetext{
${ }^{11}$ The details of the initialization of the numerical integrator for quasisynchronous rotation, taking into account even the likely orbital plane and stellar spin precession in the case of an inclined triple system, are described in Appendix A

${ }^{12} \mathrm{http}: / /$ argonaut.skymaps.info/query?
} 
Table 4. Orbital and astrophysical parameters from the joint photodynamical light curve, single-line RV curve, and ETV solution.

\begin{tabular}{|c|c|c|}
\hline \multicolumn{3}{|c|}{ Orbital elements $^{a}$} \\
\hline & \multicolumn{2}{|c|}{ Subsystem } \\
\hline & $\mathrm{Ba}-\mathrm{Bb}$ & A-B \\
\hline$P[\mathrm{~d}]$ & $8.1941 \pm 0.0007$ & $188.379 \pm 0.011$ \\
\hline$a\left[\mathrm{R}_{\odot}\right]$ & $16.21 \pm 0.16$ & $161.70 \pm 1.32$ \\
\hline$e$ & $0.0034 \pm 0.0019$ & $0.2212 \pm 0.0007$ \\
\hline$\omega[\mathrm{deg}]$ & $63.42 \pm 16.62$ & $243.93 \pm 0.66$ \\
\hline$i[\mathrm{deg}]$ & $89.776 \pm 0.198$ & $89.853 \pm 0.015$ \\
\hline$\tau[\mathrm{BJD}-2400000]$ & $57988.964 \pm 0.381$ & $58009.854 \pm 0.224$ \\
\hline$T_{0}^{b}[\mathrm{BJD}-2400000]$ & $57993.670 \pm 0.001$ & $58018.446 \pm 0.003$ \\
\hline$\Delta \Omega[\mathrm{deg}]$ & \multicolumn{2}{|c|}{$0.155 \pm 0.432$} \\
\hline$i_{\mathrm{m}}[\mathrm{deg}]$ & \multicolumn{2}{|c|}{$0.173 \pm 0.397$} \\
\hline Mass ratio $\left[q=m_{2} / m_{1}\right]$ & $0.883 \pm 0.013$ & $1.139 \pm 0.054$ \\
\hline$K_{\mathrm{A}}\left[\mathrm{km} \mathrm{s}^{-1}\right]$ & - & $23.727 \pm 0.230$ \\
\hline$\gamma\left[\mathrm{km} \mathrm{s}^{-1}\right]$ & - & $-7.113 \pm 0.230$ \\
\hline
\end{tabular}

Stellar parameters

$\begin{array}{lll}\mathrm{Ba} & \mathrm{Bb} & \mathrm{A}\end{array}$

Fractional radius $[R / a]$

Fractional luminosity [in

Relative quantities

Kepler-band]

Fractional luminosity [in

$R_{C}$-band]

Fractional luminosity [in $i^{\prime}$-band]

$\begin{array}{cc}0.0275 \pm 0.0012 & 0.0233 \pm 0.0013 \\ 0.0455 & 0.0281 \\ 0.0345 & 0.0228 \\ 0.0605 & 0.0383\end{array}$

$0.00442 \pm 0.00022$

0.9264

0.9427

Physical quantities

\begin{tabular}{lccc}
$m\left[\mathrm{M}_{\odot}\right]$ & $0.452 \pm 0.014$ & $0.399 \pm 0.012$ & $0.746 \pm 0.040$ \\
$R\left[\mathrm{R}_{\odot}\right]$ & $0.445 \pm 0.019$ & $0.378 \pm 0.024$ & $0.715 \pm 0.030$ \\
$T_{\text {eff }}[\mathrm{K}]$ & $3405 \pm 129$ & $3325 \pm 169$ & $4861 \pm 97$ \\
$L_{\text {bol }}\left[\mathrm{L}_{\odot}\right]$ & $0.0240 \pm 0.0041$ & $0.0157 \pm 0.0038$ & $0.2562 \pm 0.0298$ \\
$M_{\text {bol }}$ & $8.79 \pm 0.19$ & $9.25 \pm 0.26$ & $6.22 \pm 0.13$ \\
$\log g[\mathrm{dex}]$ & $4.80 \pm 0.04$ & $4.88 \pm 0.06$ & $4.60 \pm 0.04$ \\
$\left(M_{V}\right)_{\text {tot }}$ & & $6.57 \pm 0.14$ & \\
Distance $^{c}[\mathrm{pc}]$ & & $424 \pm 32$ & \\
\hline
\end{tabular}

Notes. ${ }^{\text {a Instantaneous, osculating orbital elements, calculated for the time of the very first integration step. }{ }^{\mathrm{b}} \text { Superior }}$ conjunction of the primary component of the given binary. (In the case of the inner 8-d binary it is equivalent to the time of mid-primary eclipse); ${ }^{c}$ Photometric distance, see the text for details.
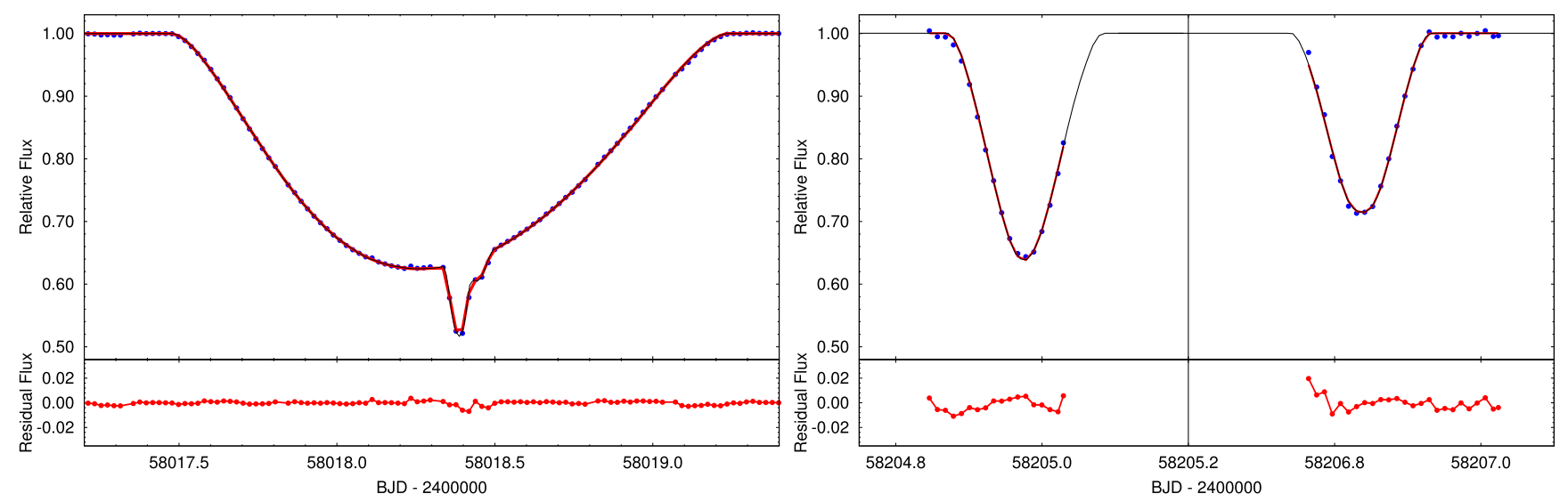

Figure 4. The great eclipses of the 'great eclipser'. Left-hand panel: The outer eclipse observed by the Kepler spacecraft; Right-hand panel: The two, separated outer eclipses, one outer orbital period (i.e. 188 d) later, observed with a ground-based telescope. The observed data are shown as blue circles. The red curve is the photodynamical model solution calculated around the time of each observation; the residuals are also shown in the bottom panels. Finally, the thin black curve represents the model solution for zero cadence time, calculated with a time step of 0.0001 of the inner binary orbital period. 

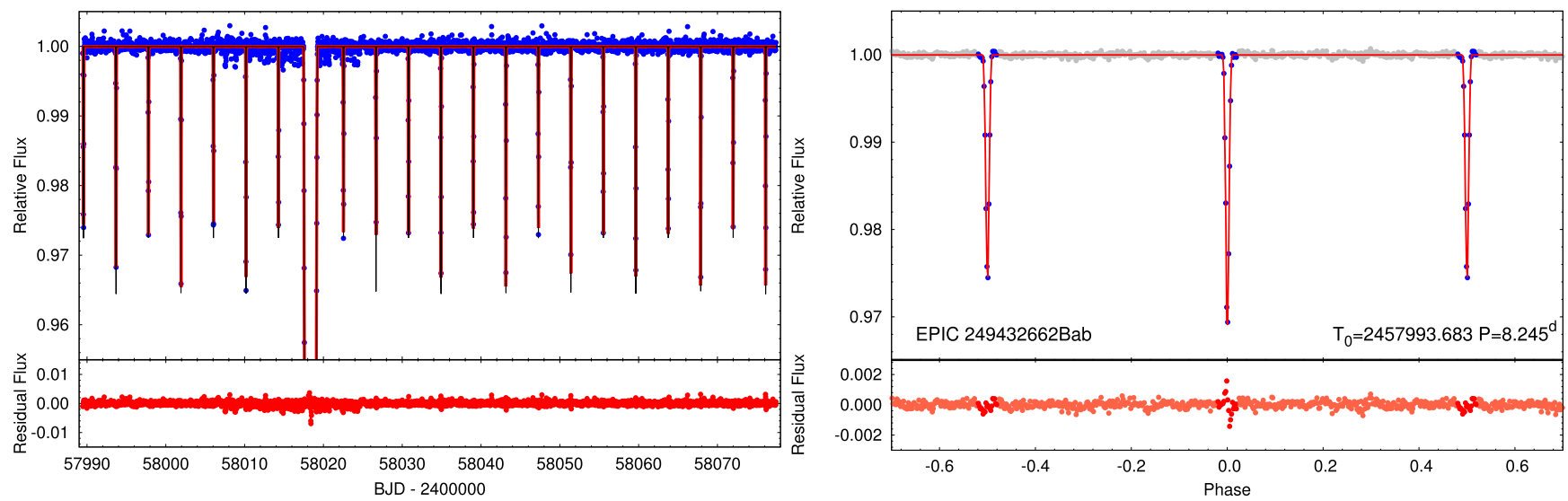

Figure 5. The shallow, 8-d binary eclipses of the great eclipser. Left-hand panel: A zoom-in on the entire K2-light curve, showing the regular, shallow eclipses of the inner binary pair; right-hand panel: The phase-folded, binned, and averaged K2-light curve of the inner binary after removing the great eclipse section from the light curve. The observed data are shown as blue and grey circles. Note, for the 'eclipses-only'-type solutions only data points within the blue phase domains were considered. The red curve is the folded, binned, and averaged light curve of the cadence-time corrected photodynamical model solution calculated at the time of each observation, the residuals of which are also shown in the bottom panels. Finally, the thin black curve (in the left-hand panel) represents the model solution for zero cadence time, calculated evenly with a time step of 0.0001 of the inner orbital period.

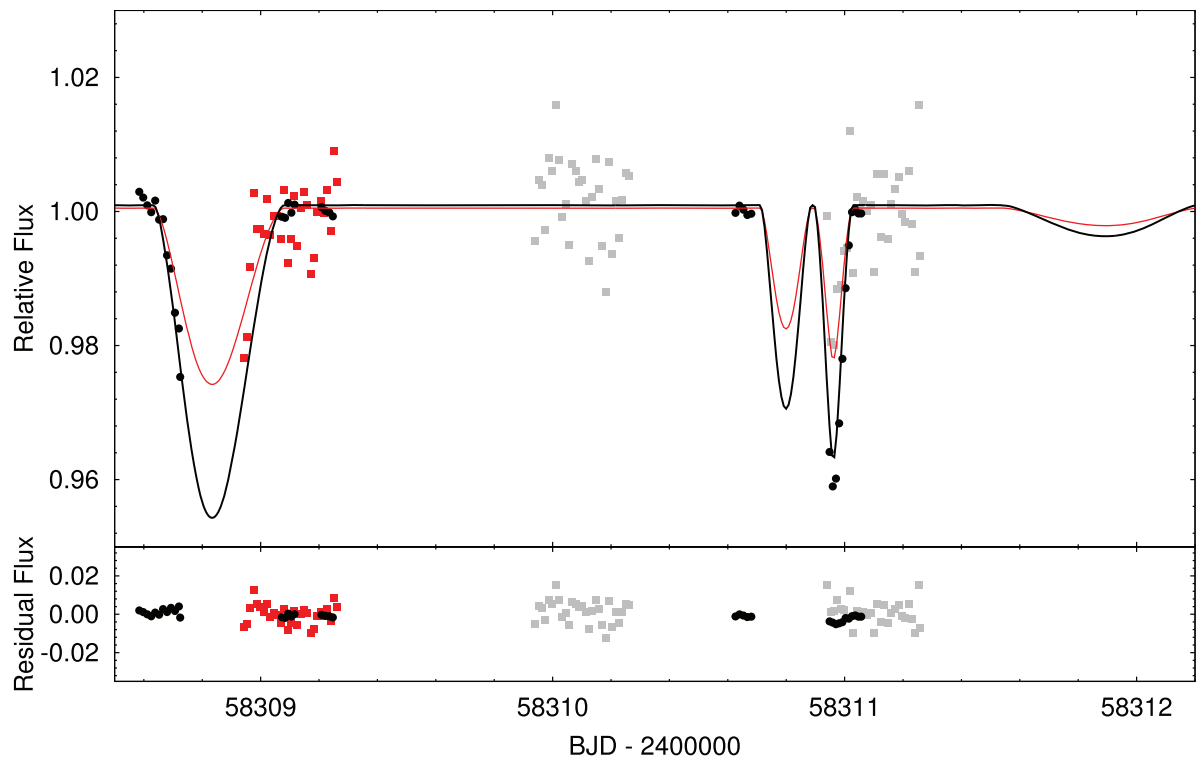

Figure 6. Secondary outer (i.e. 188-d) eclipses observed during our second ground-based follow-up campaign. Three secondary outer eclipse events of the 'great eclipser', for which the first was partially observed (around 58308.8), while the other two remained completely unobserved (including a shallow one near 58311.8), as well as a regular secondary eclipse of the inner (8-d) binary pair (the sharpest event at 58311), which was also partially observed. Red squares and black circles represent ground-based observations in $R_{\mathrm{C}}$ and $i^{\prime}$ bands, respectively, while grey squares stand for further $R_{\mathrm{C}}$-band observations that were not used for the photodynamical analysis. Red and black lines represent the photodynamical model light curves in $R_{\mathrm{C}}$ and $i^{\prime}$ bands, respectively.

$-V)=0.24 \pm 0.03$, which we translate to $A_{V}=0.75 \pm 0.09$. Thus, taking $V=15.46$ from Zacharias et al. (2013), we find a distance modulus of $8.14 \pm 0.18$. This translates to a photometric distance of $424 \pm 32 \mathrm{pc}$, which is in quite satisfactory agreement with the Gaia-determined distance of $445 \pm 7$ pc (see Table 1).

Note, however, that the use of a uniform prior for $T_{\mathrm{A}}$, instead of the spectroscopically constrained Gaussian prior, in our photodynamical analysis has resulted in an effective temperature of $T_{\mathrm{A}} \approx$ $4861 \pm 97 \mathrm{~K}$ for the outer component, star A, which is higher by about $190 \mathrm{~K}(\sim 2 \sigma)$ than the spectroscopically determined value of $4672 \pm 100 \mathrm{~K}$ (see Section 6). Despite the fact that, in principle, the spectroscopic temperature should be better determined than the photometric value (which is constrained indirectly through the different eclipse depths of the three separate photometric-band light curves), we prefer the photometric temperature, since the use of the spectroscopic value would result in a significantly lower system brightness and, therefore, a photometric distance that would be inconsistently close relative to the Gaia distance. On the other hand, however, we stress again that some caution is necessary with the currently available Gaia parallaxes. In particular, they might be rendered less accurate by systematic effects that may arise from the yet unconsidered internal 188-d motion of component A. 


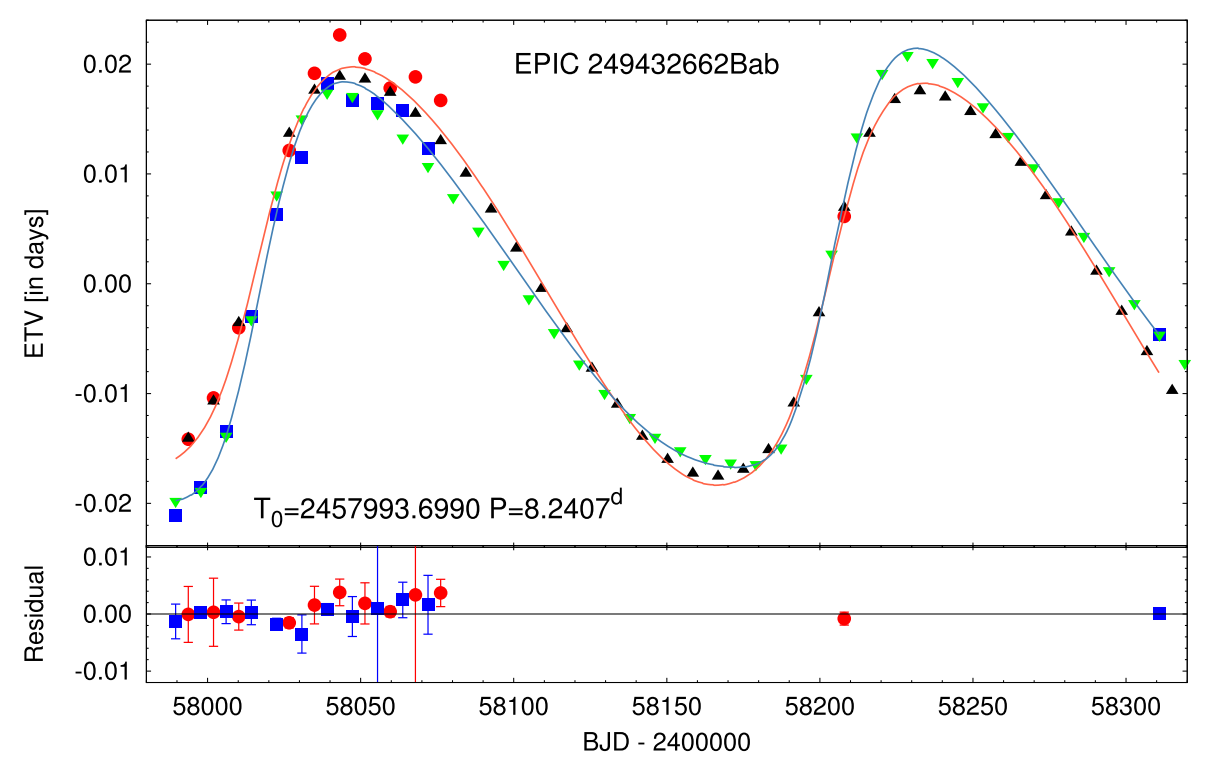

Figure 7. Eclipse timing variations of the inner binary. Upper panel: Red circles and blue squares show the primary and secondary ETVs determined from the $K 2$ and ground-based observations, while upward black and downward green triangles stand for the primary and secondary ETVs calculated from the photodynamical model, respectively. Furthermore, the thin orange and cyan lines represent the theoretical primary and secondary ETV curves obtained from a separate analytical solution (see the text for details). Bottom panel: Observed minus photodynamically modeled ETV residuals with the uncertainties of the observed ETVs. As before, red circles and blue rectangles stand for primary and secondary eclipses, respectively.

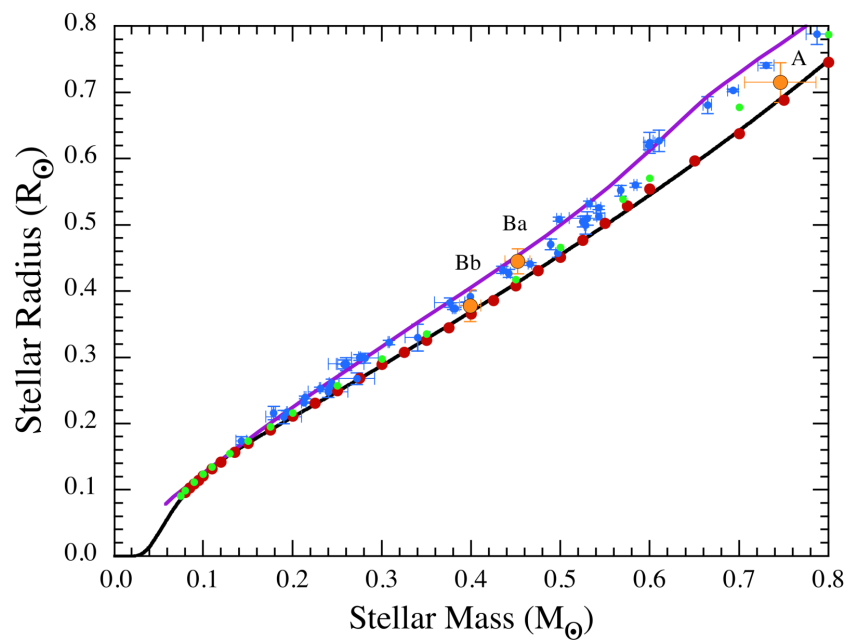

Figure 8. Comparison of 'great eclipser' masses and radii (large orange circles) compared with stellar radius versus mass relations on the lower main sequence. The red circles are models presented in Rappaport et al. (2017) for solar metallicity stars. The light green circles are taken from the Baraffe et al. (1998) results for similar stars. The solid black curve is the log-polynomial fit (equation A1 in Rappaport et al. 2017) to the model points shown in red. Blue circles with error bars are well-measured systems (see e.g. Cakirli et al. 2010; Carter et al. 2011; Kraus et al. 2011; Dittmann et al. 2017, and references therein). The purple curve is from Stassun et al. (2018) and represents the mean expected $R(M)$ value, for cool stars that are possibly thermally inflated due to their interactions in binary systems. This figure is adapted from fig. 16 of Rappaport et al. (2017).

\subsection{Orbital/dynamical properties}

Our results reveal an extremely flat triple system. The mutual inclination was found to be $i_{\text {mut }}=0.17 \pm 0.40$. Note that this narrow error bar is actually quite realistic. Our numerical integrations (in agreement with analytic perturbation theory) reveal that even a mu- tual inclination of $2^{\circ}-3^{\circ}$ would result in the rapid precession of the orbital planes, leading to significant variations in the eclipse depths of the inner binary even within the few-month-long observed data sets. Note that our numerical integration predicts a variation in the inclination angle of the 8-d binary's orbital plane of $\Delta i_{1} \approx-0.05$ over the next $2 \mathrm{yr}$.

In spite of the very good fits we obtained, we also investigated the possibility of a flat but retrograde (i. e., $i_{\text {mut }} \sim 180^{\circ}$ ) scenario for the inner versus outer orbits. For this scenario we departed from the accepted prograde solution, but (i) added $180^{\circ}$ to $\Omega_{2}$ transposing the ascending and descending nodes of the outer orbit, (ii) flagged the regular inner binary eclipse at $T_{0} \simeq 2457993.6848$ as being a secondary eclipse instead of primary one, thereby shifting the positions of the binary stars on their close orbit by $\sim 180^{\circ}$ and consequently, (iii) changed the initial phase of the secondary binary eclipse relative to the primary one by $1-\Delta \phi$, which essentially resulted in an $\sim 180^{\circ}$ difference in $\omega_{1}$. Applying these settings, we obtained a flat, but retrograde triple system for which the light curve reproduced the outer eclipse patterns rather well. On the other hand, due to the phase shift of the two inner binary stars the depth ratio of the primary and secondary eclipses reversed, resulting in disagreement with the observed light curve. Furthermore, for the dependence of the dynamical ETV-pattern on the observable argument of periastron $\left(\omega_{1}\right)$, the simulated ETV curve in the retrograde scenario also departed strongly from the observed one. ${ }^{13}$ (Note that since none of these input-parameter changes had any effect on the radial velocity curve of the outer component, the latter was indifferent in this sense.) Finally, we made some MCMC runs initiating our triple system with this parameter set, but none of these runs resulted in a suitable retrograde solution. Therefore, we can conclude with great confidence that EPIC 249432662 is a flat and prograde triple.

\footnotetext{
${ }^{13}$ The usefulness of dynamical ETV curves in deciding between prograde and retrograde solutions was also noted recently by Nemravová et al. (2016) and Brož (2017).
} 
As far as we know, there is only one other known hierarchical triple star system that has been found to be so extremely flat as the 'great eclipser'. This is the triply eclipsing system HD 181068 (KIC 05952403) in which, similar to this work, it was the analysis of the triply eclipsing light curve that led to a similar conclusion (Borkovits et al. 2013). On the other hand, however, there is a significant difference between the two systems. The outer component of HD 181068 is an evolved red giant star with a fractional radius (i.e. $R_{\mathrm{A}} / a_{2}$ ) that is larger by a factor of 25 than that in the present system $(\approx 0.138$ versus $\approx 0.005$, respectively). Therefore, in the HD 181068 system, tidal effects should have played a significant role in the dynamical evolution of the orbits, while this is not the case in the present triple. Interestingly, the shortest known outer-period Kepler-triple, KOI-126 (KIC 05897826) which, similar to EPIC 249432662, is formed by three main-sequence stars (the inner binary members are very low mass M-stars), has a significant non-zero mutual inclination $\left(i_{\text {mut }} \sim 8^{\circ}\right.$; Carter et al. 2011), while the similarly short outer period triple HD 144548 is probably flat $\left(i_{\text {mut }}\right.$ $<2^{\circ}$ ), though this latter result was inferred only indirectly from the radial velocity analysis, instead of a dynamical study (Alonso et al. 2015).

This flatness of EPIC 249432662, together with the very similar (and close to unity) inner and outer mass ratios, may imply that all three stellar components were formed sequentially from the same viscous disc. This formation scenario, which is an extension of the equal-mass, sequential binary formation mechanism, is described briefly in Tokovinin (2018).

Regarding the present-day dynamical behaviour of our triple system, the outer-to-inner period ratio of $P_{2} / P_{1}$ is $\approx 22.8$, making this triple a member of the exclusive group of 15 most compact triple stellar systems discovered amongst the Kepler EBs. As a consequence, the orbital elements are subject to significant cyclic variations due to third-body perturbations that are readily observable even during the half-year-long time-scale of the outer orbital period. The best example is the quasi-sinusoidal, $\sim 0.03$-d full-amplitude ETV curve (see Fig. 7). The ratio of the dynamical ETV amplitude relative to the light-travel-time contribution is $\mathcal{A}_{\text {dyn }} / \mathcal{A}_{\text {LTTE }} \sim 7.7$.

As a double check, we carried out an independent analysis of the ETV curves using our analytic ETV curve emulator and fitting software package described in Borkovits et al. (2015, 2016). The mathematical model was identical with the previously described one; however, for solving the inverse problem, instead of the previously employed non-linear Levenberg-Marquardt algorithm, we also used the same MCMC algorithm that was applied in our photodynamical code. For this parameter search we fixed the mutual inclination at $i_{\mathrm{m}} \equiv 0$ and, mode 'AP3' was used for modeling the dynamically forced apsidal motion of the inner binary, i.e. the period of the apsidal motion was not an additional free parameter, but rather was calculated from the formulae of the analytical perturbation theory (in first order), as was discussed in Appendix C of Borkovits et al. (2015). The orbital elements and other parameters obtained from this analytic solution are given in Table 5. For an easy comparison to the photodynamical results, we also tabulate the averages over one binary period of the same orbital elements (calculated for the very first numerically integrated complete orbit of the inner binary; see Appendix A). Furthermore, the analytical ETV solution is also plotted (as thin orange and cyan lines) in Fig. 7.

We have compared the complex light-curve+ETV +RV photodynamical results with those obtained from fits using purely analytical expressions for the ETVs. Apart from the masses, and especially the reversal of the outer mass ratio $\left(M_{A} / M_{B}\right)$ in the analytic ETV case, the orbital elements we obtain nicely match each other. We
Table 5. Comparison of the results of the purely analytical ETV solution and the photodynamical analysis.

\begin{tabular}{lcc}
\hline Parameter & Analytic ETV & Photodynamical \\
\hline$P_{1}[\mathrm{~d}]$ & $8.2304 \pm 0.0005$ & 8.2344 \\
$a_{1}\left[R_{\odot}\right]$ & $13.71 \pm 1.08$ & 16.26 \\
$e_{1}$ & $0.0055 \pm 0.0007$ & 0.0084 \\
$\omega_{1}[\mathrm{deg}]$ & $83.01 \pm 0.73$ & 84.74 \\
$i_{1}[\mathrm{deg}]$ & 89.83 & 89.78 \\
$\Omega_{1}[\mathrm{deg}]$ & 0.00 & 0.00 \\
$\tau_{1}[\mathrm{BJD}-2400000]$ & $57997.646 \pm 0.017$ & \\
$P_{2}[\mathrm{~d}]$ & $188.41 \pm 0.18$ & 188.32 \\
$a_{2}\left[R_{\odot}\right]$ & $153.70 \pm 4.94$ & 161.66 \\
$e_{2}$ & $0.172 \pm 0.019$ & 0.221 \\
$\omega_{2}[\mathrm{deg}]$ & $63.65 \pm 6.04$ & 64.06 \\
$i_{2}[\mathrm{deg}]$ & 89.83 & 89.85 \\
$\Omega_{2}[\mathrm{deg}]$ & 0.00 & 0.08 \\
$\tau_{2}[\mathrm{BJD}-2400000]$ & $58010.1 \pm 4.5$ & \\
$\left(T_{0}\right)_{2}[\mathrm{BJD}-2400000]$ & $58019.8 \pm 5.7$ & 58018.4 \\
$P_{\text {apse }}[\mathrm{yr}]$ & $33 \pm 3$ & 23.6 \\
$m_{\mathrm{A}}\left[M_{\odot}\right]$ & $0.86 \pm 0.05$ & $0.75 \pm 0.04$ \\
$m_{\mathrm{B}}\left[M_{\odot}\right]$ & $0.51 \pm 0.12$ & $0.85 \pm 0.02$ \\
\hline
\end{tabular}

stress the very solid agreement in the times of the superior conjunction of the outer orbit $\left(T_{0}\right)_{2}$ that is very strongly constrained in the joint photodynamical solution through the location and shape of the $K 2$ observed outer eclipse. By contrast, in the case of a pure ETV solution, there is no strong direct constraint on this parameter, and its value can be inferred only indirectly, and with much lesser accuracy than the orbital elements obtained from the complete photodynamical solution. These results provide further, a posteriori, confirmation of the robustness of the analytical method described in Borkovits et al. (2015).

Numerical integrations, as well as analytical computations, further reveal, however, directly non-observable, cyclic variations on both the time-scales of the inner and outer orbital periods. These are nicely illustrated below in Fig. A1 of Appendix A. (These short time-scale variations need some care in the strict interpretation of the meaning of the orbital elements tabulated in Table 4, as is discussed in Appendix A.) The third class of periodic perturbations in hierarchical triple systems are the so-called 'apse-node' time-scale perturbations, which have the longest period and highest amplitude (see e.g. Brown 1936). These include the apsidal motion of both the inner and outer orbits, which will already be observable in EPIC 249432662 in the near future with ground-based follow-up observations. Note that Fig. 7 nicely illustrates that, according to our solution, the phase displacements of the secondary eclipses of the 8-d binary relative to the primary eclipses had reversed their signs by the time of the latest outer primary eclipsing events around JD 2458206 (i.e. for now, the primary ETV curve is running below the secondary one). This is a direct consequence of the dynamical apsidal motion of the inner binary, and this reversal corresponds to that time domain when the 1-orbit-averaged $\omega_{1}$ becomes greater than $90^{\circ}$ (see the upper right panel in Fig. A1). Unfortunately, in terms of an observational verification of this model prediction, the times of only one primary and one widely separated secondary eclipse of the 8-d binary have been observed since the K2 observation. These are inconclusive as to this reversal due to the large time lapse between them. A quick and certain verification of such a reversal of the timing of the primary and secondary eclipses of the 8-d binary would require observations of a few consecutive 
Table 6. Predicted inner (8-d) eclipse times for EPIC 249432662.

\begin{tabular}{lccccc}
\hline BJD & Type & BJD & type & BJD & Type \\
-2400000 & & -2400000 & & -2400000 & \\
\hline 58422.2314 & $\mathrm{p}$ & 58549.9362 & $\mathrm{~s}$ & 58677.6706 & $\mathrm{p}$ \\
58426.3588 & $\mathrm{~s}$ & 58554.0472 & $\mathrm{p}$ & 58681.8006 & $\mathrm{~s}$ \\
58430.4714 & $\mathrm{p}$ & 58558.1773 & $\mathrm{~s}$ & 58685.9076 & $\mathrm{p}$ \\
58434.5977 & $\mathrm{~s}$ & 58562.2907 & $\mathrm{p}$ & 58690.0385 & $\mathrm{~s}$ \\
58438.7106 & $\mathrm{p}$ & 58566.4212 & $\mathrm{~s}$ & 58694.1449 & $\mathrm{p}$ \\
58442.8359 & $\mathrm{~s}$ & 58570.5379 & $\mathrm{p}$ & 58698.2768 & $\mathrm{~s}$ \\
58446.9492 & $\mathrm{p}$ & 58574.6708 & $\mathrm{~s}$ & 58702.3825 & $\mathrm{p}$ \\
58451.0739 & $\mathrm{~s}$ & 58578.7883 & $\mathrm{p}$ & 58706.5156 & $\mathrm{~s}$ \\
58455.1872 & $\mathrm{p}$ & 58582.9234 & $\mathrm{~s}$ & 58710.6208 & $\mathrm{p}$ \\
58459.3116 & $\mathrm{~s}$ & 58587.0377 & $\mathrm{p}$ & 58714.7548 & $\mathrm{~s}$ \\
58463.4249 & $\mathrm{p}$ & 58591.1726 & $\mathrm{~s}$ & 58718.8597 & $\mathrm{p}$ \\
58467.5492 & $\mathrm{~s}$ & 58595.2831 & $\mathrm{p}$ & 58722.9947 & $\mathrm{~s}$ \\
58471.6622 & $\mathrm{p}$ & 58599.4170 & $\mathrm{~s}$ & 58727.0995 & $\mathrm{p}$ \\
58475.7868 & $\mathrm{~s}$ & 58603.5255 & $\mathrm{p}$ & 58731.2350 & $\mathrm{~s}$ \\
58479.8993 & $\mathrm{p}$ & 58607.6582 & $\mathrm{~s}$ & 58735.3404 & $\mathrm{p}$ \\
58484.0244 & $\mathrm{~s}$ & 58611.7662 & $\mathrm{p}$ & 58739.4754 & $\mathrm{~s}$ \\
58488.1364 & $\mathrm{p}$ & 58615.8977 & $\mathrm{~s}$ & 58743.5824 & $\mathrm{p}$ \\
58492.2621 & $\mathrm{~s}$ & 58620.0059 & $\mathrm{p}$ & 58747.7169 & $\mathrm{~s}$ \\
58496.3734 & $\mathrm{p}$ & 58624.1365 & $\mathrm{~s}$ & 58751.8267 & $\mathrm{p}$ \\
58500.4999 & $\mathrm{~s}$ & 58628.2450 & $\mathrm{p}$ & 58755.9619 & $\mathrm{~s}$ \\
58504.6106 & $\mathrm{p}$ & 58632.3747 & $\mathrm{~s}$ & 58760.0750 & $\mathrm{p}$ \\
58508.7382 & $\mathrm{~s}$ & 58636.4835 & $\mathrm{p}$ & 58764.2127 & $\mathrm{~s}$ \\
58512.8483 & $\mathrm{p}$ & 58640.6126 & $\mathrm{~s}$ & 58772.4648 & $\mathrm{~s}$ \\
58516.9767 & $\mathrm{~s}$ & 58644.7215 & $\mathrm{p}$ & 58776.5742 & $\mathrm{p}$ \\
58521.0863 & $\mathrm{p}$ & 58648.8502 & $\mathrm{~s}$ & 58780.7129 & $\mathrm{~s}$ \\
58525.2160 & $\mathrm{~s}$ & 58652.9591 & $\mathrm{p}$ & 58784.8187 & $\mathrm{p}$ \\
58529.3251 & $\mathrm{p}$ & 58657.0879 & $\mathrm{~s}$ & 58788.9565 & $\mathrm{~s}$ \\
58533.4557 & $\mathrm{~s}$ & 58661.1964 & $\mathrm{p}$ & 58793.0606 & $\mathrm{p}$ \\
58537.5649 & $\mathrm{p}$ & 58665.3254 & $\mathrm{~s}$ & 58797.1972 & $\mathrm{~s}$ \\
58541.6959 & $\mathrm{~s}$ & 58669.4335 & $\mathrm{p}$ & 58801.3010 & $\mathrm{p}$ \\
58545.8055 & $\mathrm{p}$ & 58673.5629 & $\mathrm{~s}$ & 58805.4365 & $\mathrm{~s}$
\end{tabular}

Notes. Times are given in BJD-2400000. $p$ and $s$ refer to primary and secondary eclipses, respectively.

(or nearly consecutive) primary and secondary eclipses. In order to help prepare for the encouraged future follow-up observations, we tabulate the predicted times of minima for the next $1 \mathrm{yr}$ in Table 6.

Regarding the forthcoming outer eclipses, according to our model, the 2018 fall event should have been the longest great eclipse at least over the following decade (see the blue curve in Fig. 9). As one can see, this outer primary eclipse was expected to have had an extremely long duration of almost $2.3 \mathrm{~d}$ between 2018 September 30 and 2018 October 2. This epoch, however, was unfortunately unfavourable for ground-based follow-up observations due to the source's proximity to the Sun. In Fig. 9 we also plot the previous two great (primary) eclipse events (black and green curves) together with the next upcoming one (red curve). For the convenience of observers, we list the predicted time intervals for the next few outer primary and secondary eclipses in Table 7.

In order to test the long-term stability of this triple, we carried out 1-million-yr-long numerical integrations with the same integrator that was also used during the photodynamical analysis. Irrespective of whether we used a simple three-body point-mass model, or included tidal effects, we did not detect any secular variations in the orbital elements. Therefore, we can conclude that, not surprisingly, the present configuration of EPIC 249432662 is stable over the nuclear time-scale of star A.

\section{SUMMARY AND CONCLUSIONS}

In this work we report the discovery of a remarkable triple star system with an inner 8-d M-star binary orbiting a $\mathrm{K}$ star in a 188d mildly eccentric orbit. Both orbital planes are nearly perfectly aligned with us the observers. The motion of one of the stars in the 8-d binary can conspire to nearly cancel its relative motion across the outer $\mathrm{K}$ star during opposition, thereby producing very long eclipses (up to $2 \mathrm{~d}$ ).

We have carried out a comprehensive photodynamical analysis of the photometric, RV, and ETV data sets. This analysis has led to a good assessment of all the system parameters (see Table 4), including the three constituent masses, orbital parameters, and the conclusion that the two orbital planes are flat to within $\sim 0.2^{\circ}$. We also checked the more quantitative photodynamical solution against fits to analytic approximations to the ETV curves in order to 'calibrate' how well they work.

The masses of the two M stars in the 8-d binary are determined with about 5 percent precision $(1.5 \sigma)$, which is sufficient to make them interesting comparison stars to test against stellar evolution models of lower-main sequence stars. As with the relatively few previously well-determined systems, the radii are somewhat, but significantly, larger than models from the latest stellar evolution codes indicate.

The system has a substantially low ratio of outer to inner periods. This leads to a number of different dynamical interactions, which are described in the text. Perhaps the most observationally dramatic of these is the large-amplitude ETV curve that we measure, and model both analytically and with a direct 3-body integration.

The accuracy with which the system parameters are known can be improved by future observations. We have demonstrated that small telescopes used by amateurs can readily observe the deep and broad outer 3rd-body eclipses, as well as even the 8-d binary eclipses. In this regard, we provide a list of times for future outer eclipses as well as eclipses of the 8-d binary. We encourage such observations over the next couple of observing seasons. According to our projected orbital solution, the very next upcoming outer eclipse is expected to be the longest in duration, and the deepest in amplitude, for at least the forthcoming decade.

\section{ACKNOWLEDGEMENTS}

T. B. acknowledges the financial support of the Hungarian National Research, Development and Innovation Office - NKFIH Grant OTKA K-113117. We thank Bruce Gary for reducing the data from the JBO observations. M.H.K., M.R. O., H.M.S., and I. T. acknowledge Allan R. Schmitt for making his light-curve examining software lctools freely available. We are thankful for the fruitful discussions with Sz. Csizmadia (DLR, Berlin) and I. B. Bíró (Baja Astronomical Observatory, Hungary). W. D. C. and M. E. acknowledge support from NASA grants NNX16AJ11G and 80NSSC18K0447 to The University of Texas at Austin. This work was performed in part under contract with the California Institute of Technology/Jet Propulsion Laboratory funded by NASA through the Sagan Fellowship Program executed by the NASA Exoplanet Science Institute. This paper includes data collected by the $K 2$ mission. Funding for the $K 2$ mission is provided by the NASA Science Mission directorate. Some of the data presented in this paper were obtained from the Mikulski Archive for Space Telescopes (MAST). STScI is operated by the Association of Universities for Research in Astronomy, Inc., under NASA contract NAS5-26555. Support for MAST for non-HST data is provided by the NASA Office of 


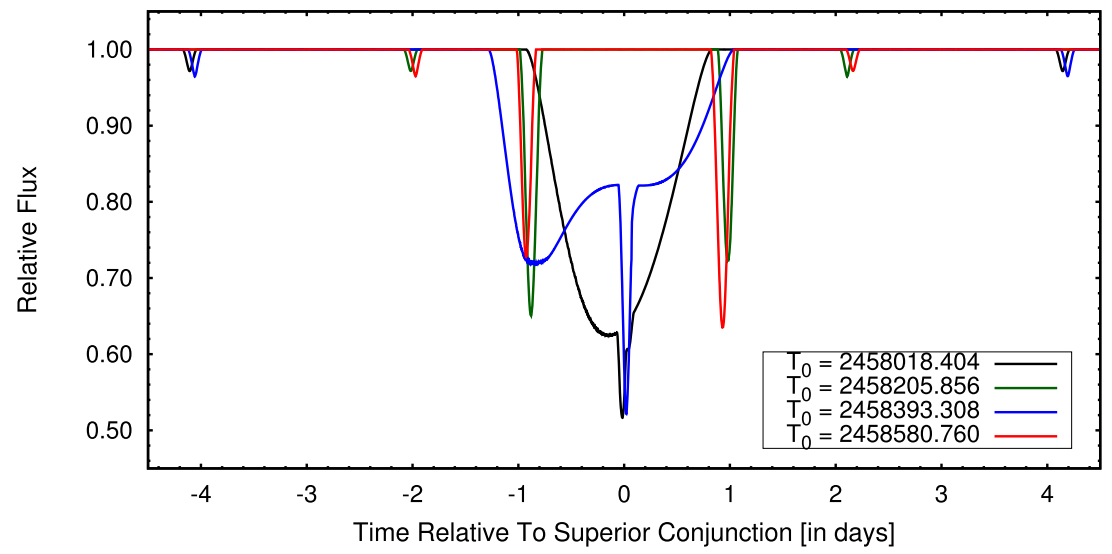

Figure 9. Past and future great eclipses of EPIC 249432662. The photodynamical model light curve of the Kepler-spacecraft (black) and ground-based (green) observed previous two primary outer eclipses, as well as the predicted forthcoming two events (blue and red, respectively). The closest regular, inner eclipses to the outer superior conjunctions provide a key to understanding the qualitative characteristics of the individual eclipses. As one can see, in the black and blue cases the outer superior conjunction occurs at the time of an inner (8-d binary) eclipse. Therefore, due to the coplanar configuration, the backward component of the inner binary (i.e. the one which is eclipsed during the inner eclipse) has a very small projected velocity relative to the eclipsed third component during its transit, which results in extremely long eclipse durations. The main difference between the black and blue curves arises from the fact that the role of the two inner binary members is exchanged. A comparison of the amplitudes of the black and blue inner eclipses that are separated by $\sim 4 \mathrm{~d}$ from the outer eclipses reveals that in the case of the great eclipse observed by $K 2$ the corresponding 8-d binary eclipse was a primary event, i.e. the more massive star $B a$ was the relatively slowly moving component. By contrast, in the forthcoming event (which should have occurred in the fall of 2018, after the submission of this paper), shown with the blue curve, the less massive $B b$ component with its physically faster orbital motion will move even more slowly relative to the outer stellar component, resulting in an almost 2.5-d-long great eclipse. On the other hand, due to its smaller stellar radius, this long-lasting event will have a lower amplitude than the corresponding portion of the $K 2$ event. Furthermore, as one can see, at the maximum phase of the eclipse (i.e. during the spike), when both components of the inner binary eclipse the third star, the fading will reach the same magnitude as in the case of the $K 2$ eclipse. In contrast to these two great eclipses, in the case of the green (past) and red (future) events, the outer superior conjunction occurs near to the quadrature of the inner binary and, therefore, the two binary members eclipse the third star separately and relatively quickly. Note that the roles of the two inner (8-d) binary members are also reversed for these two events.

Table 7. Predicted outer eclipse times over the next $1000 \mathrm{~d}$ for EPIC 249432662.

\begin{tabular}{lcc}
\hline First event & Last event & Type \\
\hline 58392.0 & 58394.3 & $\mathrm{p}$ \\
58496.9 & 58499.4 & $\mathrm{~s}$ \\
58579.7 & 58581.8 & $\mathrm{p}$ \\
58683.0 & 58685.5 & $\mathrm{~s}$ \\
58767.2 & 58768.7 & $\mathrm{p}$ \\
58871.5 & 58873.8 & $\mathrm{~s}$ \\
58954.7 & 58956.8 & $\mathrm{p}$ \\
59057.7 & 59060.2 & $\mathrm{~s}$ \\
59141.8 & 59143.4 & $\mathrm{p}$ \\
59244.1 & 59248.4 & $\mathrm{~s}$ \\
59329.6 & 59331.6 & $\mathrm{p}$ \\
59432.3 & 49434.8 & $\mathrm{~s}$ \\
59516.9 & 59518.3 & $\mathrm{p}$ \\
\hline
\end{tabular}

Notes. Times are given in BJD-2400000, and stand for the beginning of the first fading and the end of the last fading; $p$ and $s$ refer to the primary and secondary outer (188-d) eclipses, respectively.

Space Science via grant NNX09AF08G and by other grants and contracts. The authors wish to extend special thanks to those of Hawai'ian ancestry on whose sacred mountain of Maunakea we are privileged to be guests. Without their generous hospitality, the Keck observations presented herein would not have been possible.

\section{REFERENCES}

Alonso R., Deeg H. J., Hoyer S., Lodieu N., Palle E., Sanchis-Ojeda R., 2015, A\&A, 584, L8
Armstrong D. et al., 2012, A\&A, 545, L4

Auvergne M. et al., 2009, A\&A, 506, 411

Baraffe I., Chabrier G., Allard F., Hauschildt P. H., 1998, A\&A, 337, 403

Barensten G., Cardosos J. V. d. M., 2018, Astrophysics Source Code Library,record ascl.soft03005B

Borkovits T., Csizmadia Sz., Hegedüs T., Bíró I. B., Sándor Zs., Opitz A., 2002, A\&A, 392, 895

Borkovits T., Forgács-Dajka E., Regály Zs., 2004, A\&A, 426, 951

Borkovits T., Csizmadia, Sz., Forgács-Dajka E., Hegedüs T., 2011, A\&A, 528, A53

Borkovits T. et al., 2013, MNRAS, 428, 1656

Borkovits T., Rappaport S., Hajdu T., Sztakovics J., 2015, MNRAS, 448, 946

Borkovits T., Hajdu T., Sztakovics J., Rappaport S., Levine A., Bíró I. B., Klagyivik P., 2016, MNRAS, 455, 4136

Borkovits T. et al., 2018, MNRAS, 478, 5135

Borucki W. J. et al., 2010, Sci, 327, 977

Brown E. W., 1936, MNRAS, 97, 62

Brown T. M. et al., 2013, PASP, 125, 1031

Brož M., 2017, ApJS, 230, 19

Bryson S. T. et al., 2010, ApJ, 713, L97

Cakirli, Ö., Ibanuglu C., Dervisoglu A., 2010, RMxAA, 46, 363

Carter J. A. et al., 2011, Science, 331, 562

Castelli F., Kurucz R. L., 2004, preprint (arXiv:astro-ph/0405087)

Chambers K. C. et al., 2016, preprint (arXiv:1612.05560)

Christiansen J. L. et al., 2018, AJ, 155, 57

Chubak C., Marcy G., Fischer D. A., Howard A. W., Isaacson H., Johnson J. A., Wright J. T., 2012, preprint (arXiv:1207.6212)

Claret A., Bloemen S., 2011, A\&A, 529, A75

Collins K. A., Kielkopf J. F., Stassun K. G., Hessman F. V., 2017, AJ, 153, 77

Conroy K. E., Prša A., Stassun K. G., Orosz J. A., Fabrycky D. C., Welsh W. F., 2014, AJ, 147, 45 
Cutri R. M. et al., 2013, wise.rept, 1C

David T. J. et al., 2018, AJ, 155, 222

Derekas A. et al., 2011, Science, 332, 216

Dittmann J. A. et al., 2017, ApJ, 836, 124

Eggleton P. P., Kiseleva-Eggleton L., 2001, ApJ, 562, 1012

Eggleton P. P., Kiseleva L., Hut P., 1998, ApJ, 499, 853

Endl M., Cochran W. D., 2016, PASP, 128, 94502

Fabrycky D., Tremaine S., 2007, ApJ, 669, 1298

Fehlberg E., 1974, NASA Technical Report R-432

Flower P. J., 1996, ApJ, 469, 355

Ford E. B., 2005, AJ, 129, 1706

Giménez A., Garcia-Pelayo J. M., 1983, Ap\&SS, 92, 203

Haas M., Hackstein M., Ramolla M., Drass H., Watermann R., Lemke R., Chini R., 2012, AN, 333, 706

Hajdu T., Borkovits T., Forgács-Dajka E., Sztakovics J., Marschalkó G., Benkő J. M., Klagyivik P., Sallai M. J., 2017, MNRAS, 471, 1230

Han E. et al., 2017, AJ, 154, 100

Hełminiak K. G., Ukita N., Kambe E., Kozłowski S. K., Pawłaszek R., Maehara H., Baranec C., Konacki M., 2017, A\&A, 602, A30

Howard A. W. et al., 2010, ApJ, 721, 1467

Howell S. B. et al., 2014, PASP, 126, 398

Irwin J. B., 1959, AJ, 64, 149

Kipping D. M., Schmitt A. R., Huang X., Torres G., Nesvorný D., Buchhave L. A., Hartman J., Bakos G. Á., 2015, ApJ, 813, 14

Kiseleva L. G., Eggleton P. P., Mikkola S., 1998, MNRAS, 300, 292

Kolbl R., Marcy G. W., Isaacson H., Howard A. W., 2015, AJ, 149, 18

Kraus A. L., Tucker R. A., Thompson M. L., Craine E. R., Hillenbrand L. A., 2011, ApJ, 728, 48

Lindegren L. et al., 2018, A\&A, 616, A2

Loeb A., Gaudi B. S., 2003, ApJ, 588, L117

Lucy L. B., 1967, Zeitschrift für Astrophisik, 65, 89

Maoz D., Mannucci F., Nelemans G., 2014, ARA\&A, 52, 107

Marsh T. R., Armstrong D. J., Carter P. J., 2014, MNRAS, 445, 309

Masuda K., Uehara Sh., Kawahara H., 2015, ApJ, 806, L37

Maxwell M., Kratter K. M., 2018, ApJ, 854, 44

Naoz S., Fabrycky D. C., 2014, ApJ, 793, 137

Nemravová J. A. et al., 2016, A\&A, 594, A55

Orosz J. A., 2015, ASPC, 496, 55

Perets H. B., Fabrycky D. C., 2009, ApJ, 697, 1048

Portegies Zwart S., van den Heuvel E. P. J., van Leeuwen J., Nelemans G., 2011, ApJ, 734, 55

Prša A., Zwitter T., 2005, ApJ, 628, 426

Ransom S. M. et al., 2014, Nature, 505, 520

Rappaport S. et al., 2017, MNRAS, 467, 2160

Shappee B. J., Thompson T. A., 2013, ApJ, 766, 64

Shibahashi H., Kurtz D. W., 2012, MNRAS, 422, 738

Skrutskie M. F. et al., 2006, AJ, 131, 1163

Slawson R. W. et al., 2011, AJ, 142, 160

Stassun K. G. et al., 2018, AJ, 156, 102

Tokovinin A., 2014b, AJ, 147, 87

Tokovinin A., 2017, ApJ, 844, 103

Tokovinin A., 2018, AJ, 155, 160

Tokovinin A., Thomas S., Sterzik M., Udry S., 2006, A\&A, 450, 681

Torres G., 2010, AJ, 140, 1158

Tout C. A., Pols O. R., Eggleton P. P., Han Z., 1996, MNRAS, 281, 257

Tull R. G., MacQueen P. J., Sneden C., Lambert D. L., 1995, PASP, 107, 251

van Kerkwijk M. H., Rappaport S., Breton R., Justham S., Podsiadlowski, Ph., Han Z., 2010, ApJ, 715, 51

Vanderburg A., Johnson J., 2014, PASP, 126, 948

Villanueva S., Jr., Gaudi B. S., Pogge R. W., Eastman J. D., Stassun K. G., Trueblood M., Trueblood P., 2018, PASP, 130, 5001

Yee S. W., Petigura E. A., von Braun K., 2017, ApJ, 836, 77

Yu L. et al., 2018, AJ, 156, 22

Zacharias N., Finch C. T., Girard T. M., Henden A., Bartlett J. L., Monet D. G., Zacharias M. I., 2013, ApJS, 145, 44

\section{APPENDIX A: SOME DETAILS OF THE \\ NUMERICAL INTEGRATOR AND THE INITIALIZATION OF THE ORBITAL ELEMENTS}

The numerical three-body integrator that is built into our code was originally developed for studying tidal effects on the dynamics of hierarchical triple stellar systems. Hence, it integrates not only the equations of orbital motion of the three bodies, including tidal and rotational effects with arbitrarily directed stellar spin axes, but also, simultaneously, the Eulerian equations of rotation of the stellar components, regarding the stars as deformable bodies. ${ }^{14}$ The dependent variables of the integrator are the six Cartesian coordinates and velocity components of the first two Jacobian vectors (i.e. $\vec{\rho}_{1}$ which connects the center of mass of the two components of the inner binary, directed from the primary to the secondary, and $\vec{\rho}_{2}$ which connects the center of mass of the inner binary with the outer, third component), and also the Eulerian angles and their first derivatives, describing the stellar rotations for each star individually. The details of the integrator were given in Borkovits et al. (2004), and we consider here only some relevant notes on the conversion between the orbital elements and the Cartesian inputs and outputs of our integrator in the case of such a compact, dynamically active triple system.

As is well known, in the case of either third-body or tidal perturbations, the orbital motion of a binary component will no longer be pure Keplerian and, therefore, the usual orbital elements will not retain their exact meaning. There are, however different treatments to preserve and generalize the meanings of orbital elements for perturbed systems. In our photodynamical code we do this in the most usual and intuitive way, i.e. we use the so-called 'osculating' orbital elements. This means that the orbital elements (used only as input parameters) are converted to Cartesian coordinates and velocities at the given epoch in the same way as for the unperturbed case. One should keep in mind, however, that osculating orbital elements at later instants of time will differ from the initial one, as is nicely illustrated in Fig. A1. This requires some extra care when one intends to compare the photodynamical results with some observational results obtained at significantly different epochs.

Furthermore, Fig. A1 illustrates some other issues, both theoretical and practical ones. For example, as one can see (in the upper row), for very small binary eccentricities, the argument of periastron is very weakly determined. In other words, the orientation of the major axis of the orbital ellipse might be subject to large variations within very short intervals. This fact reveals a principal weakness in using the osculating orbital elements. In order to illustrate this, let us consider a point mass orbiting a body that is a permanently deformed into an oblate spheroid (e.g. rotationally deformed) on an exactly circular orbit. In this case, as long as the extra (tidal) force remains radial and constant in time, the motion remains strictly circular. On the other hand, calculating the osculating orbital elements of the point mass, one would infer a small, but definitely non-zero, eccentricity and a continuously rotating argument of periastron due to the fact that the orbiting point mass is located permanently at the periastron point of the osculating ellipse. (This question was discussed, and some suggestions for resolving the problem were given,

\footnotetext{
${ }^{14}$ We stress that general relativistic effects, including relativistic precession, have not yet been included into the equations of motions. Note, however, that in our system the relativistic precession rates are $\left(\dot{\omega}_{1}\right)_{\mathrm{GR}} \approx 19^{\prime \prime} 3 \mathrm{yr}^{-1}$ and $\left(\dot{\omega}_{2}\right)_{\mathrm{GR}} \approx 00^{\prime} .17 \mathrm{yr}^{-1}$, which can safely be ignored relative to the dynamical apsidal motion rate of $\left(\dot{\omega}_{1}\right)_{\text {dyn }} \approx 15.3 \mathrm{yr}^{-1}$
} 

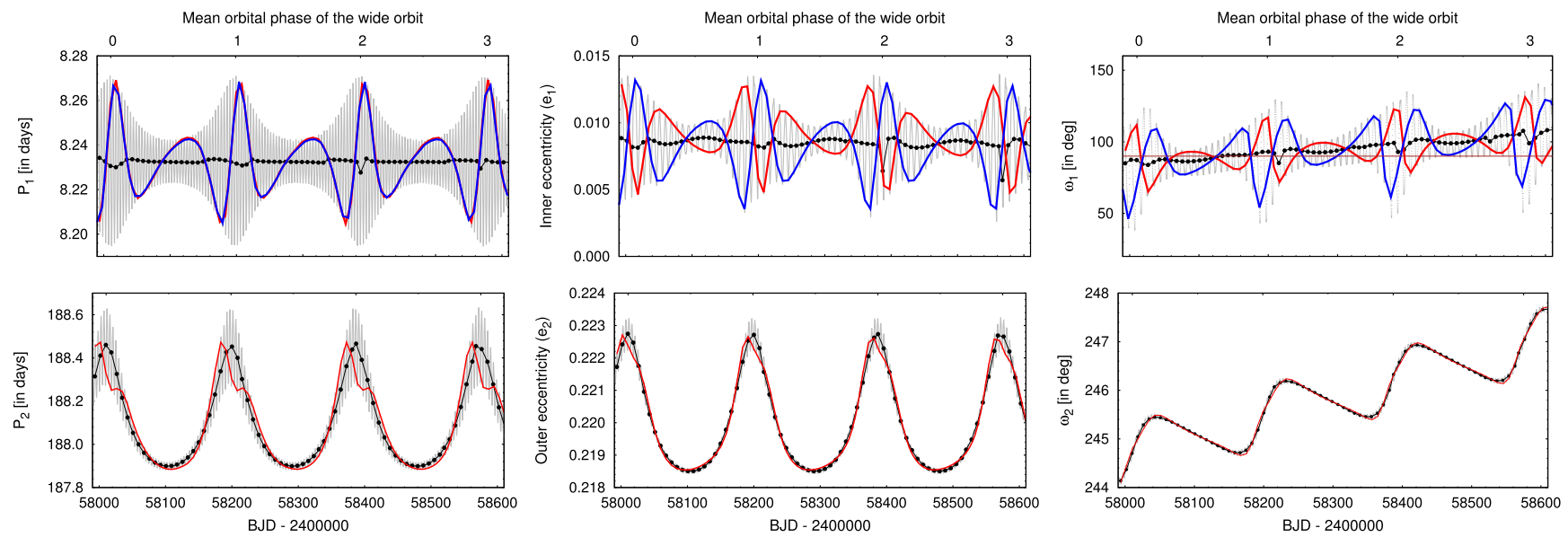

Figure A1. Variations of the instantaneous (osculating) anomalistic periods, eccentricities, and (observable) arguments of periastron of the inner and outer binaries (grey curves in all panels), obtained via numerical integration, together with the one-inner-orbit averages (black). Furthermore, the red and blue lines connect the instantaneous orbital elements sampled at those integration points that are closest to the primary and secondary mid-eclipse points of the inner binary, respectively. (Note, for the outer orbit the red and blue lines coincide almost perfectly; therefore, we plot only the red one.) The brown horizontal line at $\omega_{1}=90^{\circ}$ (in the upper right panel) helps to identify the domain where the phase displacement of the inner secondary eclipse relative to photometric phase 0.5 reverses its sign. (See the text for details.)

e.g. in Kiseleva et al. 1998 and Borkovits et al. 2002.) Fortunately, as we found, the use of the alternative orbital elements $e \cos \omega$ and $e \sin \omega$ (or, more or less equivalently, the displacement of the secondary eclipse from photometric phase 0.5 ) during the initialization of the numerical integrator avoids this problem, therefore, it has no practical consequence.

By contrast, the large fluctuations in the osculating instantaneous anomalistic period of the inner pair (top left panel) have very unpleasant consequences for the speed of convergence of the MCMC parameter search; this is especially true when the ETV curves are included into the fitting process. The reason is as follows. It is well known that the ETV is extraordinarily sensitive to the mean sidereal period since the amplitude of each ETV point is proportional to the product of the error in that period and the cycle number, growing linearly in time. Here the following problem arises. The relevant input parameter of the photodynamics code is the instantaneous anomalistic period; however, the ETV is governed chiefly by the mean sidereal period. The instantaneous anomalistic period depends strongly on the orbital elements of both orbits, and also on the accurate locations of the stars along their orbits (i.e. the orbital phases), not to mention their masses. Therefore, small changes in any of these parameters at each trial step in the fitting process lead to a significant mis-tuning of the mean sidereal period, thereby resulting in large $\chi_{\text {ETV }}^{2}$ values. This can slow significantly the convergence of the MCMC chains despite the fact that the fits were seemingly good anyway.

The ideal solution to the mismatch between the two different types of periods would be to try to convert the mean sidereal period to the instantaneous anomalistic period at the epoch when the numerical integrator is initialized and, thereby, the former would be used as an input parameter. To find such an analytical relation would require, however, unrealistically large and time-consuming efforts, and thus we applied another simple, heuristic solution, as follows. During each trial step, our code first calculates the numerical ETV points (see Appendix B below) and then adjusts this numerical ETV to the observed one with a two-parameter linear least-squares fit using the difference in the epoch $\left(T_{0}\right)$ and mean sidereal period $\left(P_{\mathrm{s}}\right)$ as free parameters. Then these differences are considered as approximate corrections to the initial instantaneous anomalistic orbital period and the initial phase-term of the inner orbit, and they are therefore simply added to the previous input values. Then, the same process is repeated once again (we found that two iterations satisfactorily constrain these parameters), and the resultant initial parameters are used for the subsequent trial step of the given chain. This includes the calculation not only of the model light curves and RV curve, but also the re-calculation of the model ETVs.

Finally, for the sake of completeness, we discuss another issue regarding the initialization of certain parameters at each trial step. This is the question of the synchronous rotation of any of the stars. Regarding the orbital motion of a binary system, it is well-known that in the presence of any third-body perturbations, the orbit cannot remain permanently exactly circular, and therefore not exactly synchronous. Furthermore, unless the perturber is exactly located in the binary's orbital plane, there should also occur a precession of that plane. As a consequence, one also can expect that the equatorial plane of the binary stars and the outer orbital plane will no longer be continuously aligned. Therefore, due to an inclined perturber, some stellar spin precession should also occur (for further discussion, see e. g. Eggleton, Kiseleva \& Hut 1998; Kiseleva et al. 1998; Borkovits et al. 2004).

Taking into account these considerations in our numerical integrator (and, therefore, in the complete photodynamical treatment), we apply the following iterative process during the initialization of the spin parameters of a star flagged as a 'quasi-synchronous rotator':

(i) First the code takes the stellar rotational equatorial plane to be identical with the orbital plane, setting the Eulerian angles of stellar rotation equal to the corresponding orbital elements ${ }^{15}$ as $\theta=i, \phi=$

\footnotetext{
${ }^{15}$ Orbital elements $i, \Omega$ are also practically Eulerian angles, describing the orientation of the orbital plane relative to the base of the coordinate system, i.e. the tangential plane of the sky.
} 
$\Omega$, and setting the uni-axial spin angular velocity equal to the orbital angular velocity calculated at periastron passage, i.e. $\omega_{z^{\prime}}=w_{\text {per }}$.

(ii) Using these initial values, the code calculates the instantaneous Cartesian force components.

(iii) Using the known perturbing force, the code calculates the instantaneous time derivatives of the angular orbital elements, and sets $\dot{\theta}=i$ and $\dot{\phi}=\dot{\Omega}$. Similarly, the perturbed orbital angular velocity (at periastron passage) is calculated as well and, the uni-axial spin angular velocity (and, similarly, the time derivative of the third Eulerian angle, $\dot{\psi})$ are set accordingly.

(iv) Finally, the same process from item (ii) is repeated twice again.

\section{APPENDIX B: NUMERICAL ETV CURVE GENERATION}

Both photometric fluxes and radial velocities at any given time are direct outputs of the light-curve and RV curve emulator code for any sets of the initial model parameters, and therefore, can be readily compared to their observational counterparts. However, this is not so for the model times of eclipse minima. In order to obtain the necessary mid-eclipse times and, therefore, to obtain the model ETV points from the numerical integration of the equations of motion of the triple system, we applied the following quick and approximate, but sufficiently accurate method.

The numerical integrator calculates the Jacobian coordinates and velocities of the three stars at any given observed mid-eclipse time $\left(t_{\mathrm{obs}}\right)$. Then, these coordinates and velocities were converted back to instantaneous osculating orbital elements, as well as the corresponding instantaneous eccentric anomaly, $\mathcal{E}_{1}\left(\mathrm{t}_{\mathrm{obs}}\right)$, which we write more simply as $\mathcal{E}_{1, \text { obs }}$, where the subscript ' 1 ' refers to the inner binary, as opposed to the outer orbit. By the use of the same osculating, instantaneous orbital elements, the theoretical true anomaly at the moment of the model mid-eclipse was also calculated as

$f_{1, \mathrm{calc}}=\mp \frac{\pi}{2}-\omega_{1} \pm \frac{e_{1} \cos \omega_{1} \cos ^{2} i_{1}}{\sin ^{2} i_{1} \mp e_{1} \sin \omega_{1}}$, where the last term on the right-hand side is a first-order approximation of the exact expression given by Giménez \& Garcia-Pelayo (1983) describing the very weak inclination-angle dependence of the mid-eclipses for eccentric orbits. The upper signs refer to primary eclipses, while the lower signs are for the secondary ones. (Note that we define $\omega$ as the argument of periastron of the secondary component relative to the primary.) Then, we convert this true anomaly at the time of a model eclipse to the corresponding eccentric anomaly via the familiar relation

$\mathcal{E}_{1, \text { calc }}=2 \arctan \left(\sqrt{\frac{1-e_{1}}{1+e_{1}}} \tan \frac{f_{1, \text { calc }}}{2}\right)$,

From $\mathcal{E}_{1, \text { calc }}$ and $\mathcal{E}_{1, \text { obs }}$ the model mid-eclipse times, relative to the observed ones, can be calculated easily as

$$
\begin{aligned}
t_{\text {model }}= & t_{\mathrm{obs}}-\frac{m_{\mathrm{A}}}{m_{\mathrm{AB}}} \frac{Z_{2}}{c} \\
& +\frac{P_{1}}{2 \pi}\left[\mathcal{E}_{1, \text { calc }}-\mathcal{E}_{1, \mathrm{obs}}-e_{1}\left(\sin \mathcal{E}_{1, \text { calc }}-\sin \mathcal{E}_{1, \mathrm{obs}}\right)\right] .
\end{aligned}
$$

Here, the last term in the first row of the right-hand side stands for the light-travel time contribution, where $Z_{2}$ denotes the $Z$ coordinate of the second Jacobian vector, i.e. the radius vector directed from the centre of mass of the inner pair to the outer component.

We tested the accuracy of this fast, but approximate, calculation of the model eclipse times by comparing these values with those calculated from the model light curve by the same method as the observed eclipse times were calculated. We found good correspondance between the two types of simulated ETV data, down to a scale of $\sim 10^{-4} \mathrm{~d}$.

This paper has been typeset from a $\mathrm{T}_{\mathrm{E}} \mathrm{X} / \mathrm{L} \mathrm{T} \mathrm{E} \mathrm{X}$ file prepared by the author. 